\title{
The Unmanned Aerial Vehicle Routing and Trajectory Optimisation Problem, a taxonomic review
}

\author{
Walton Pereira Coutinho ${ }^{\mathrm{a}}$, Maria Battarra ${ }^{\mathrm{b}}$, Jörg Fliege ${ }^{\mathrm{a}}$ \\ ${ }^{a}$ University of Southampton, University Road, Southampton, SO17 1BJ, United Kingdom \\ $\{w . p . c o u t i n h o, j . f l i e g e\} @$ soton.ac.uk \\ ${ }^{b}$ University of Bath, Claverton Down, Bath, BA2 7AY, United Kingdom \\ m.battarra@bath.ac.uk
}

\begin{abstract}
Over the past few years, Unmanned Aerial Vehicles (UAVs) have become more and more popular. The complexity of routing UAVs has not been fully investigated in the literature. In this paper, we provide a formal definition of the UAV Routing and Trajectory Optimisation Problem (UAVRTOP). Next, we introduce a taxonomy and review recent contributions in UAV trajectory optimisation, UAV routing and articles addressing these problems, and their variants, simultaneously. We conclude with the identification of future research opportunities.
\end{abstract}

Keywords: unmanned aerial vehicles, routing, trajectory optimisation, literature review, taxonomy

\section{Introduction}

Unmanned Aerial Vehicles (UAVs) are aircraft that do not need a human pilot on board. In general, these vehicles are either controlled by an embedded computer or by a pilot operating a remote control. Drones, remote controlled helicopters and unmanned gliders are examples of UAVs. Gliders differ from UAVs were first developed in the 1920s to support military operations in which the presence of human pilots was either impossible or too dangerous (Beard \& McLain, 2012; Keane \& Carr, 2013). However, UAVs have recently become very popular for logistics and surveillance applications (Tsourdos et al., 2010).

A report from the National Purchase Diary has shown that sales of drones increased by $224 \%$ in twelve months from April 2015, reaching a total of 200 million dollars (NPD, 2016). Due to being able to embed several transmitters, sensors and photographic equipment, UAVs can be used in a large range of applications. Successful cases have been reported in, for example, aerial reconnaissance (Ruzgiené et al., 2015), aerial forest fire detection (Yuan et al., 2015), target observation (Rysdyk, 2006), traffic monitoring and management (Kanistras et al., 2013), online commerce (Wang et al., 2017), geographical monitoring (Uysal et al., 2015), scientific data collection (Stöcker et al., 2015), meteorological sampling (Elston et al., 2014) and disaster assessment and response (Quaritsch et al., 2010; Xu et al., 2014; Nedjati et al., 2016). In Hayat et al. (2016), several applications of UAV networks are reviewed. The use of UAVs for 3D mapping is surveyed in Nex \& Remondino (2013). A literature review about the applications of UAVs in humanitarian relief is provided by Bravo \& Leiras (2015). More examples of the growing applications of UAVs are presented in Rao et al. (2016). 
The academic routing community has acknowledged the interest of companies and organisations in adopting UAVs in their operations. A recent example is the approach of combining UAVs and trucks for distribution activities by dispatching drones from trucks for the last mile distribution within city centres (Ha et al., 2015; Murray \& Chu, 2015; Wang et al., 2017). It has been shown that this solution can reduce truck travel time, and the corresponding $\mathrm{CO}_{2}$ emissions, by up to 50\%. The UAV Task Assignment Problem (UAVTAP), which is closely related to the UAV routing problem, consists of optimising the assignment of a set of UAVs to a set of tasks subject to mission constraints (Khamis et al., 2015). A growing body of literature appeared on the UAVTAP in the last decade, e.g., Ramirez-Atencia et al. (2016), Wang et al. (2015), Hu et al. (2015a), Thi et al. (2012), Alidaee et al. (2010) and Edison \& Shima (2011). However, the UAV routing and task assignment literatures have often neglected constraints due to the flight dynamics of the UAVs. Finding feasible trajectories for UAVs in a routing problem is a complex task, but it is necessary to ensure the feasibility of the UAVs routes. For some real-world applications involving more complex UAV systems, such as unmanned gliders and fixed-wing vehicles, the definition of routes must be coupled to the design of flyable trajectories, otherwise the assigned routes might become inefficient or even infeasible for these UAVs.

Most of the UAVs used for civil applications present a low flight autonomy. Therefore, it is important for UAV routing algorithms to properly model battery life. According to Fügenschuh \& Müllenstedt (2015), this can be achieved by integrating the UAVs' dynamics with routing. As mentioned by the authors, for powered UAVs, a proper modelling of the actual fuel consumption must include, for instance, the current weight, the altitude, the speed and climb/descent rate, which are usually modelled by flight dynamics.

Zhang et al. (2012) consider a problem where a UAV must visit a set of targets. However, after reaching a predetermined distance from a target the UAV must then adjust its flight attitude (i.e., its orientation) in order to perform a payload delivery. After the delivery, the UAV must complete an escape manoeuvre and prepare for the next delivery. According to Zhang et al. (2012), routing and trajectory optimisation must be integrated in order to ensure the safety of the vehicle and the feasibility of trajectories.

The computation of trajectories for UAVs has been widely studied in the aerospace engineering and optimal control literature (Yang et al., 2016). The Trajectory Optimisation (TO) problem consists of finding a control history of a given vehicle, that minimises a scalar performance index (for example, flight time or fuel consumption) while satisfying constraints on the kinematics (position, velocity and acceleration) and the dynamics (forces and moments) of the vehicle (Betts, 1998). A trajectory is generally associated with a set of Equations of Motion (EOMs) that describe the relationship between the spatial and the temporal changes to the system. The TO problem is closely related to the Optimal Control (OC) problem (Betts, 2001).

The problem named Path Planning (PP) consists of finding a flyable path for a UAV visiting a given sequence of waypoints (targets) in a two-dimensional (2D) or three-dimensional (3D) space without considering the vehicle's dynamics. According to Gasparetto et al. (2015), PP is a geometric problem, because it is defined as finding a geometric path regardless any specified time law. In turn, TO consists of assigning a time law to a controlled geometric path.

More complex variants of the PP problem including, for instance, wind and motion constraints, require 
substantial simplifications and assumptions to be solved heuristically (Kunchev et al., 2006; Rathinam \& Sengupta, 2007). The books by Tsourdos et al. (2010) and Beard \& McLain (2012) provide good overviews of PP algorithms for UAVs. On the other hand, high fidelity TO models (i.e., using more accurate physical models) have been developed for aircraft and spacecraft (Raivio et al., 1996; Conway, 2010; Fisch, 2011; García-Heras et al., 2014; Colasurdo et al., 2014). These models are currently solved by OC techniques. An overview of OC methods for TO is provided in Betts $(1998,2001)$.

The field of TO has however not considered routing decisions: given a set of ordered waypoints, it is possible to find a feasible trajectory for a generic UAV, but it is not clear in the literature if the sequence of waypoints is appropriate. For example, for a gliding vehicle (i.e., with no onboard thrust) a given waypoint sequence might be infeasible in terms of flight dynamics. Given a fleet of UAVs, it is an open question how to combine routing and trajectory decisions in a single optimisation problem. As far as the authors are aware, there is not a survey summarising the literature about routing and trajectory optimisation for UAVs.

Research about integrated routing and TO problems seems to be still fragmented. One of the main contributions of this paper is introducing the UAV Routing and Trajectory Optimisation Problem (UAVRTOP). We believe that integrating TO and routing in a single optimisation problem is a key research challenge in adopting UAVs for real world applications.

The purpose of this survey is to present the UAVRTOP, highlighting approaches already proposed in the literature and providing a direction for further research. We introduce a taxonomy, that is able to identify the key components of routing and TO problems, as well as highlight assumptions and simplifications commonly adopted in the literature.

The remainder of this paper is organised as follows. In Section 2, we formally define the UAVRTOP. In Section 3, a background on TO problems is provided. The same is done in Section 4 for vehicle routing problems. In Section 5, a taxonomy of UAV routing and TO problems is provided. An application of the proposed taxonomy to a selected number of papers is demonstrated in Section 6 . This section continues with an analysis of the results obtained from the taxonomic review. In Section 7, we discuss future research opportunities.

\section{The UAV routing and trajectory optimisation problem}

In this section, we formally define the UAV Routing and Trajectory Optimisation Problem (UAVRTOP), the problem in which a fleet of UAVs has to visit a set of waypoints assuming generic kinematics and dynamics constraints. Wind conditions, collision avoidance between UAVs and obstacles can also be incorporated in the model.

\subsection{A mathematical formulation for the UAVRTOP}

In the following, we assume a fleet $C$ of UAVs is available at the launching site 0 . Let $G=(V, A)$ be a graph, where the set $V$ represents all the waypoints that need to be visited by the UAVs and $A$ represents the set of arcs between waypoints. In addition, let $0^{\prime}$ represent the landing site. The cost of using a vehicle $k \in C$ is $F_{k}$. The parameters (e.g., mass, wing area, aerodynamics coefficients) of the UAV $k$ travelling between $i$ and $j$ are stored in the vector $\mathbf{p}_{i j k}$. Note that these parameters may change 
a UAV is a vector fully defining the position, orientation and velocity of the vehicle in some coordinate system (alternative state representations will be described in Section 3).

For simplicity, we recall $\mathbf{y}_{i j k}\left(t_{i j k}\right) \in \mathbb{R}^{n_{y}^{k}}, n_{y}^{k} \in \mathbb{Z}$, the state variable of the UAV $k$ travelling between waypoints $i$ and $j$ at time $t_{i j k} \in \mathbb{R}$. Similarly, the control variables model the inputs that are given to the physical systems in order to achieve a desired trajectory. Typical control variables for UAVs are the thrust (the impulse given by the UAV engine, if any), the roll angle, a.k.a. bank angle (which banks the aircraft to change its horizontal flight direction), and the angle-of-attack (which is related to how much lift the aircraft's wing generate). We define $\mathbf{u}_{i j k}\left(t_{i j k}\right) \in \mathbb{R}^{n_{u}^{k}}, n_{u}^{k} \in \mathbb{Z}$, the control variables for a UAV $k$ flying on arc $(i, j)$ at time $t_{i j k} \in \mathbb{R}$.

The physical laws governing the UAV $k$ travelling between the waypoints $i$ and $j$ at time $t_{i j k}$ are referred as system dynamics. In general terms, the system dynamics can be expressed by a set of EOMs in the form of a system of Ordinary Differential Equations (ODEs) as follows:

$$
\dot{\mathbf{y}}_{i j k}=\mathbf{f}_{k}\left(\mathbf{y}_{i j k}\left(t_{i j k}\right), \mathbf{u}_{i j k}\left(t_{i j k}\right), \mathbf{p}_{i j k}, t_{i j k}\right) \forall i, j \in V, \forall k \in C
$$

The functions $\mathbf{f}_{k}, \forall k \in C$, in the right hand side of the EOMs (1), represent the relationship between the variables and parameters with the derivatives over time of the state variables (here denoted by " *").

State and control variables have to be specified for a time instant to initialise the ODEs. In what follows, we assume that the initial conditions need to be specified at time $t=0$. It is also reasonable to assume that only the control variables need to be optimised since the values of the states can be determined, provided an initial condition and the evolution of the controls over time.

Let $w_{k}($.$) be a function computing the cost of using UAV k$ along an arbitrary trajectory. The routing cost for a UAV $k$ to travel between waypoints $i$ and $j$ can be computed as:

$$
\int_{t_{i j k}^{o}}^{t_{i j k}^{f}} w_{k}\left(\mathbf{y}_{i j k}\left(t_{i j k}\right), \mathbf{u}_{i j k}\left(t_{i j k}\right), \mathbf{p}_{i j k}, t_{i j k}\right) \mathrm{d} t_{i j k} .
$$

The variables $t_{i j k}^{o}$ and $t_{i j k}^{f}$ represent the initial and final flight times of the UAV $k$ travelling between waypoints $i$ and $j$ such that $t_{i j k} \in\left[t_{i j k}^{o}, t_{i j k}^{f}\right]$.

Bounds on the state and control variables are usually imposed by a given UAV technology. We denote $\mathbf{y}_{i j k}^{l b}$ and $\mathbf{y}_{i j k}^{u b}$ the lower and upper bounds on the state variables $\mathbf{y}_{i j k}\left(t_{i j k}\right)$ of the UAV $k$ travelling in an $\operatorname{arc}(i, j)$ for all $t_{i j k} \in \mathbb{R}$, respectively. Similarly, $\mathbf{u}_{i j k}^{l b}$ and $\mathbf{u}_{i j k}^{u b}$ represent the lower and upper bounds of the control variables $\mathbf{u}_{i j k}\left(t_{i j k}\right)$ of the $\mathrm{UAV} k$ travelling on $\operatorname{arc}(i, j)$ for all $t_{i j k} \in \mathbb{R}$. We also assume lower and upper bounds on the operational constraints, here denoted as $\mathbf{g}_{i j k}^{l b}$ and $\mathbf{g}_{i j k}^{u b}$.

According to our assumption on the initial conditions, the initial flight time from the launching point must be defined as $t_{0 j k}^{o}=0, \forall j \in V, \forall k \in C$. Let $\overline{\mathbf{y}}_{o}$ and $\overline{\mathbf{u}}_{o}$ represent predetermined initial conditions. Thus, the initial state and control variables can be defined as $\mathbf{y}_{0 j k}\left(t_{0 j k}^{o}\right)=\overline{\mathbf{y}}_{o}$ and $\mathbf{u}_{0 j k}\left(t_{0 j k}^{o}\right)=\overline{\mathbf{u}}_{o}$, respectively, if UAV $k$ departs from the launching point.

Let us define the following binary variables:

$$
x_{i j k}= \begin{cases}1, & \text { if UAV } k \text { flies directly from waypoint } i \text { to } j \\ 0, & \text { otherwise. }\end{cases}
$$


Hereafter, we will describe the optimisation problem defined by Equations (4-19). This formulation is a conceptual model created for describing the UAVRTOP in mathematical terms. The objective function (4) minimises the sum of the fixed cost of using a UAV, the routing cost of flying between waypoints $i$

and $j$ and a measure of the quality of the trajectories at the end points of each arc $(i, j)$. Non desirable features at the end points of the UAVs' trajectories can be penalised in the objective function by means of the functions $\phi_{k}\left(\mathbf{y}_{i j k}\left(t_{i j k}^{f}\right), \mathbf{u}_{i j k}\left(t_{i j k}^{f}\right), \mathbf{p}^{i j k}, t_{i j k}^{f}\right)$. Such undesirable characteristics may include, e.g., sharp flight angles, prohibited flight speeds and noise levels (Vanderbei, 2001; Zhang et al., 2012). Constraints (5) and (6) ensure that every waypoint is visited exactly once and that, if a UAV arrives at a waypoint $l \in V$, it must also depart from $l$. Constraints (7) make sure that each UAV departs from the launching point 0 and lands in $0^{\prime}$, if the UAV $k$ is used. Constraints (8) ensure that the UAVs' dynamics are preserved if $\operatorname{arc}(i, j)$ is used in a solution. In a similar way, Constraints (9-11) make sure the bounds on the state variables, control variables and operational constraints $\left(\mathbf{g}_{i j k}\left(\mathbf{y}_{i j k}\left(t_{i j k}\right), \mathbf{u}_{i j k}\left(t_{i j k}\right), \mathbf{p}_{i j k}, t_{i j k}\right)\right)$ are respected for every arc $(i, j)$ and for every UAV $k$ if these are travelled in the obtained solution. These and (13) ensure that the final state and control variables at every arc $(i, j)$ visited by UAV $k$ is linked to the state and control variables of its subsequent $\operatorname{arc}(j, l)$ if waypoints $i, j$ and $l$ are visited by UAV $k$ in this order. Constraints (14) preserve the continuity of the time variable $t_{i j k}, \forall i, j \in V$, along the UAV's $k$ trajectory for all $k \in C$. Constraints (15) and (16) provide the initial states and controls for every UAV departing from the launching point. Finally, Constraints (17-19) define the domain of the variables.

The UAVRTOP can be modelled as follows:

$$
\begin{array}{ll}
\min & \sum_{k \in C} \sum_{i \in V} F_{k} x_{0 i k} \\
& +\sum_{k \in C} \sum_{(i, j) \in A}\left\{\int_{t_{i j k}^{o}}^{t_{i j k}^{f}} w_{k}\left(\mathbf{y}_{i j k}\left(t_{i j k}\right), \mathbf{u}_{i j k}\left(t_{i j k}\right), \mathbf{p}_{i j k}, t_{i j k}\right) \mathrm{d} t_{i j k}\right\} x_{i j k} \\
& +\sum_{k \in C} \sum_{(i, j) \in A} \phi_{k}\left(\mathbf{y}_{i j k}\left(t_{i j k}^{f}\right), \mathbf{u}_{i j k}\left(t_{i j k}^{f}\right), \mathbf{p}^{i j k}, t_{i j k}^{f}\right) x_{i j k} \\
\text { s.t. } \quad \sum_{k \in C} \sum_{i \in V \cup\{0\}} x_{i j k}=1, \forall j \in V \\
\quad \sum_{i \neq j} x_{i j k}-\sum_{i \neq j} x_{j i k}=0, \forall j \in V, \forall k \in C \\
& \sum_{i \in V} x_{0 i k}=\sum_{i \in V} x_{i 0^{\prime} k} \leq 1, \forall k \in C \\
& \dot{\mathbf{y}}_{i j k}=\mathbf{f}_{k}\left(\mathbf{y}_{i j k}\left(t_{i j k}\right), \mathbf{u}_{i j k}\left(t_{i j k}\right), \mathbf{p}_{i j k}, t_{i j k}\right) x_{i j k}, \forall i, j \in V, \forall k \in C \\
& \mathbf{g}_{i j k}^{l b} x_{i j k} \leq \mathbf{g}_{i j k}\left(\mathbf{y}_{i j k}\left(t_{i j k}\right), \mathbf{u}_{i j k}\left(t_{i j k}\right), \mathbf{p}_{i j k}, t_{i j k}\right) \leq \mathbf{g}_{i j k}^{u b} x_{i j k}, \\
& \forall i, j \in V, \forall k \in C \\
& \mathbf{y}_{i j k}^{l b} x_{i j k} \leq \mathbf{y}_{i j k}\left(t_{i j k}\right) \leq \mathbf{y}_{i j k}^{u b} x_{i j k}, \forall i, j \in V, \forall k \in C \\
& \mathbf{u}_{i j k}^{l b} x_{i j k} \leq \mathbf{u}_{i j k}\left(t_{i j k}\right) \leq \mathbf{u}_{i j k}^{u b} x_{i j k}, \forall i, j \in V, \forall k \in C \\
& \mathbf{y}_{j l k}\left(t_{j l k}^{o}\right) x_{i j k}=\mathbf{y}_{i j k}\left(t_{i j k}^{f}\right) x_{i j k} x_{j l k}, \forall i, j, l \in V, \forall k \in C \\
& \mathbf{u}_{j l k}\left(t_{j l k}^{o}\right) x_{i j k}=\mathbf{u}_{i j k}\left(t_{i j k}^{f}\right) x_{i j k} x_{j l k}, \forall i, j, l \in V, \forall k \in C \\
& \\
& \\
& \\
& \\
&
\end{array}
$$




$$
\begin{aligned}
& t_{j l k}^{o} x_{i j k}=t_{i j k}^{f} x_{i j k} x_{j l k}, \forall i, j, l \in V, \forall k \in C \\
& \mathbf{y}_{0 j k}\left(t_{0 j k}^{o}\right)=\overline{\mathbf{y}}_{o} x_{0 j k}, \forall j \in V, \forall k \in C \\
& \mathbf{u}_{0 j k}\left(t_{0 j k}^{o}\right)=\overline{\mathbf{u}}_{o} x_{0 j k}, \forall j \in V, \forall k \in C \\
& x_{i j k} \in\{0,1\}, \forall i, j \in V, \forall k \in C \\
& \mathbf{u}_{i j k}\left(t_{i j k}\right) \in \mathbb{R}^{n_{u}^{k}}, \forall i, j \in V, \forall k \in C \\
& t_{i j k}, t_{i j k}^{o}, t_{i j k}^{f} \in \mathbb{R} . \forall i, j \in V, \forall k \in C
\end{aligned}
$$

\section{The trajectory optimisation problem}

TOPs are a special case of OC problems determining the trajectory of a system (e.g., vehicles such as spacecraft, aircraft, UAVs) while minimising a measure of performance and satisfying a set of boundary (initial and final) conditions, path constraints and system dynamics.

The origin of OC problems dates to as early as the 17th century when Johann Bernoulli proposed the Brachistochrone problem (Ross, 2009), one of the first problems in calculus of variations. One of the first applications of the calculus of variations to the control of flying vehicles was presented by Robert Goddard in "A method of reaching extreme altitudes" (Goddard, 1919), where the objective was to determine the minimum initial mass of a ground-based rocket necessary to achieve a given altitude. OC methods are a classical tool in the computation of spacecraft trajectories, e.g., for interplanetary travel and satellite transfer orbits around Earth (Conway, 2010; Colasurdo et al., 2014).

Usually, system dynamics are modelled by a set of EOMs that can be nonlinear and discontinuous. six degrees of freedom (6DOF) EOMs are composed by translational equations (containing forces, position, velocity, acceleration, etc.) and rotational equations (containing moments, angular velocities, angular acceleration, etc.). Under simplifying assumptions, 6DOF EOMs can be decoupled into three degrees of freedom (3DOF) EOMs, see, e.g., Stengel (2004) and Fisch (2011). Usual state variables in 6DOF EOMs are the position vector, velocity, pitch angle, pitch rate, weight and flight path angle. In the 3DOF case, the state vector can represent, for instance, the position, velocity, flight path angle and yaw angle of the vehicle.

Solving a Trajectory Optimisation Problem (TOP) for an aircraft consists of generating the inputs for the aircraft's control system so as to perform a desired set of manoeuvres. A TOP takes as input the dynamic constraints of the aircraft and outputs time-indexed states and controls such as positions, velocities and accelerations.

Other difficulties can be added to the problem if one considers that the boundary conditions depend on unknown variables or if the dynamics of the vehicles change over time. In this cases, TOPs can be divided into two or more phases in order to properly model the changes in the operational or physical characteristics of the vehicles. A phase can be defined as a segment of a trajectory in which the dynamical system remains unchanged. Phases can be described by their own boundary conditions, system of differential equations, operational constraints and time events. Finally, all phases can be linked or not depending on the behaviour of the dynamical system.

Aircraft TO models have gained much popularity over the last decades. For instance, Schultz \& Zagalsky (1972) present solutions for several fixed endpoint aircraft TOPs using calculus of variations. In 
Raivio et al. (1996), a nonlinear programming-based method is proposed to compute optimal trajectories for a descending aircraft. Fisch (2011) presents a high fidelity optimisation framework for the computation of air race trajectories under safety requirements. García-Heras et al. (2014) compare several OC methods for the TO of cruise flight with fixed arrival time. Finally, Delahaye et al. (2014) present a survey of mathematical models for the computation of aircraft trajectories.

OC methods for UAVs are similar to those of full size aircraft, and therefore similar system dynamics can be used for both types of planes. On the other hand, new challenges are introduced when specific into determining successful flight plans. Therefore, algorithms that are capable of tackling the UAVs' particularities while developing flight plans must be developed.

\subsection{Direct and indirect methods for trajectory optimisation problems}

Two main classes of numerical methods became very popular for solving TOPs, these being, direct and indirect methods. The so-called direct methods rely on the discretisation of a infinite-dimensional OC problem into a finite-dimensional optimisation problem. This strategy is commonly known as "discretise, then optimise". In a direct single shooting method, for example, the controls are discretised on a fixed grid using an arbitrary parametrisation scheme. The next step of this method consists of solving a nonlinear programming problem in order to find an optimal vector of parameters. The indirect methods consist of determining necessary optimality conditions for an OC problem and then using a discretisation method to solve the resulting equations. Indirect methods generally apply an "optimise, then discretise" strategy. In an indirect single shooting method, for example, the resulting optimality conditions consist of a boundary value problem, which can be solved by means of a simple single shooting algorithm (Betts, 2001).

Several sophisticated algorithms have been developed for solving TOPs. Reviewing such works is considered beyond the scope of this paper. More information about algorithms for OC and TO can be found, for example, in the papers by Stryk \& Bulirsch (1992), Betts (1998), Ross (2009), Wang (2009) and Rao (2014); and, the books by Bryson (1975), Bertsekas (1979), Betts (2001), Bryson (2002) and Kirk (2012).

\subsection{The UAV path planning problem}

Using the notation defined by Latombe (1991), the basic PP problem can be defined as follows. Let $\mathcal{A}$ be an object (a robot) moving in a workspace $\mathcal{S}$ (e.g., in an Euclidean space $\mathcal{S}=\mathbb{R}^{n}, n=2$ or 3 ). A set of obstacles $\mathcal{B}_{1}, \ldots, \mathcal{B}_{m}$ is assumed to be distributed over $\mathcal{S}$. The problem consists in, given initial and final configurations (position and orientation) for $\mathcal{A}$, find a path in $\mathcal{S}$ that avoids collisions with the objects $\mathcal{B}_{1}, \ldots, \mathcal{B}_{m}$. It has been shown that this problem is $\mathcal{N} \mathcal{P}$-hard if the velocity of the object $\mathcal{A}$ is unbounded and no rotation is considered (Reif \& Sharir, 1994). For Gasparetto et al. (2015), a path planning problem consists of finding a collision-free path among an environment from an initial point to a final goal. For example, YongBo et al. (2017) studied a path planning problem with obstacles in three dimensions. In the literature, the terms PP and motion planning are used almost interchangeably (Barraquand \& Latombe, 1991). Both problems have gained much popularity over the years. Figure 1 shows the number of publications by year on UAV PP problems. 


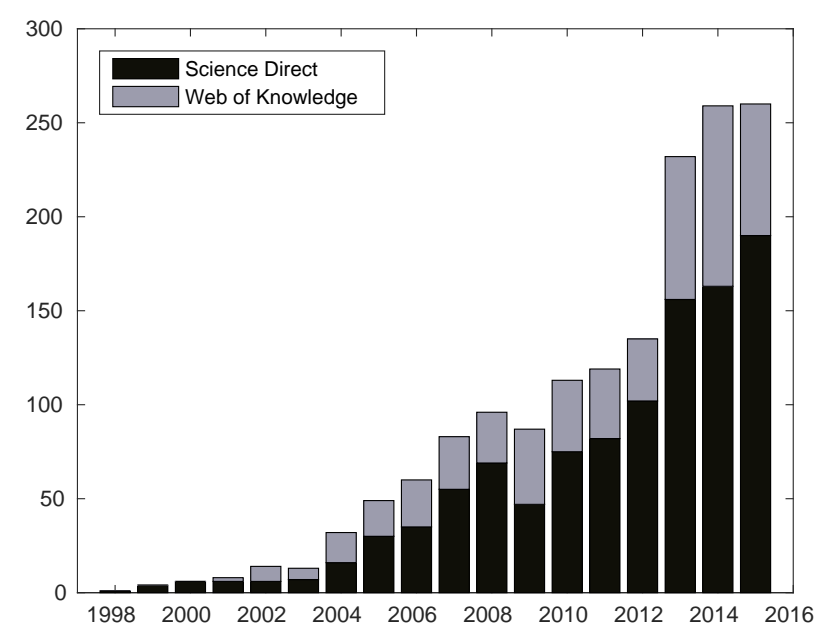

Figure 1: Number of published papers by year on PP problems.

PP algorithms can be classified into discrete and continuous methods. In the former, the workspace $\mathcal{S}$ is transformed into a graph through discretisation. Conventional heuristics or exact shortest path algorithms are then used to find a path between a given initial configuration and a final configuration. The output for discrete methods are usually polygonal paths, i.e., paths with no curvature constraints. Therefore, in the case of UAVs these paths need to be further refined. Continuous methods represent $\mathcal{S}$ by using a continuous function. Tsourdos et al. (2010), for instance, employed attraction fields to represent the desired endpoints and repulsive fields to represent obstacles in order to produce a collision-free UAV path.

Problems integrating UAV routing and PP have been studied before, see, for example, Manyam et al. (2015), Ho \& Ouaknine (2015), Enright et al. (2015), Sundar \& Rathinam (2014) and Levy et al. (2014). Under simplifying assumptions, a PP problem can be modelled as a network problem and standard shortest path techniques can be used. A common assumption is that the UAV can be modelled as a Dubin's vehicle (Medeiros \& Urrutia, 2010). A Dubin's vehicle has a limited turning angle and is restricted to move forward, therefore it can be a good representation for some types of UAVs. This simplification is very popular specially for modelling rotary-wing aircraft such as quadcopters. However, for most fixed-wing UAVs, the Dubin's assumption might not be suitable due to their complicated dynamics. The reader is referred to Tsourdos et al. (2010) for more details on UAVs PP methods.

Most algorithms for UAV PP have originated from adaptations of existing algorithms for robot PP. However, we do not intend to survey all PP algorithms as it has already been done in other articles, e.g., Kunchev et al. (2006), Goerzen et al. (2009), Galceran \& Carreras (2013) and Yang et al. (2016).

\section{The vehicle routing problem}

The Vehicle Routing Problem (VRP) is a very well known problem in operational research and combinatorial optimisation. In the VRP, routes must be assigned to a set of vehicles that must serve a set of customers such that the total cost of the operation is minimised. Its classical variant is called Capacitated Vehicle Routing Problem (CVRP), where a load capacity is assigned to each vehicle.

The CVRP can be formally defined as follows. A set of vertices $V=\{0, \ldots, n\}$ and a set of arcs $A$ connecting these vertices are given. Each vertex represents a customer with demand $d_{i}, i \in V \backslash\{0\}$. 
A value $c_{i j}$ is assigned to each arc $(i, j) \in A$ representing the travel cost between two customers. Let $C=\{1, \ldots, m\}$ be a set of homogeneous vehicles with capacity $Q$. Here we denote the vertex $i=0$ representing the depot (launching site). The CVRP consists of finding a minimum cost set of $m$ routes starting and ending at the depot such that all customers are visited exactly once, all customers' demands are satisfied and the capacity of the vehicles are respected. The CVRP is well known to be $\mathcal{N} \mathcal{P}$-hard. More information about the VRP and its variants can be found, e.g., in Golden \& Assad (1988), Cordeau et al. (2007), Golden et al. (2008), Toth \& Vigo (2002), Eksioglu et al. (2009), Lahyani et al. (2015) and Braekers et al. (2016).

The m-TSP is closely related to the VRP. In the m-TSP, $m$ minimum cost tours starting at the depot must be found such that every vertex in $V \backslash\{0\}$ is visited exactly once. The m-TSP can be reduced to the CVRP if all vehicles are considered to have infinite capacity. An extensive literature review on models and algorithms for the m-TSP is presented by Bektas (2006).

The VRP and the m-TSP have been widely studied for terrestrial applications, but with the development of new technologies, such as unmanned vehicles, new variants of these problems are gaining interest among the scientific community. The problem of routing an aerial vehicle is more complex than the VRP because it combines the combinatorial characteristics of the VRP with the complexity of dealing with the system dynamics of UAVs (i.e., flight dynamics, battery life, wind conditions).

\subsection{UAV task assignment problem}

The UAV Task Assignment Problem (UAVTAP) consists of finding an optimal assignment of UAVs to a set of tasks. Often, the UAVs have different characteristics and the tasks depend on the nature of the application. It has been shown that this problem is $\mathcal{N} \mathcal{P}$-hard (Alidaee et al., 2010). Due to the quick development of UAV technology, new challenging assignment problems arise every day and many algorithms have been developed to address the new challenges. Figure 2 shows the number of publications by year in UAVTAPs, the $y$ axis corresponds to the number of publications found in Science Direct and Web of Knowledge databases. One can observe that this field of research has gained attention of the scientific community. A detailed literature review about algorithms for multi-robot Task Assignment (TA) problems can be found in Khamis et al. (2015).

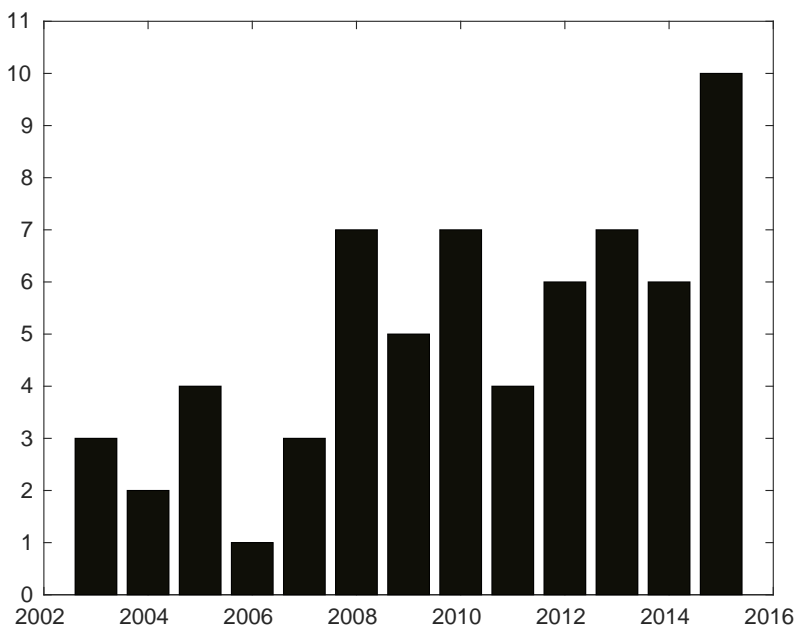

Figure 2: Number of published papers by year on TA problems. 
The UAVTAP shares some characteristics with the VRP. Many examples in the literature support this claim. One can cite, for instance, TA with time windows (Karaman \& Inalhan, 2008), multi-depot (launching points) (Darrah et al., 2012), task allocation with resource constraints (Kim et al., 2015), TA with flexible demand (Alidaee et al., 2011), real-time and dynamic assignment (Kim et al., 2007; Lin et al., 2013), time-dependent TA (Kingston \& Schumacher, 2005), and finally, TA under uncertainty (Alighanbari \& How, 2008; Hu et al., 2015a). However, the UAVTAP differs from the VRP by allowing, for example, multiple visits and subtours. In addition, new features may also be introduced, e.g., the possibility of heterogeneous UAVs to perform multiple operations at the same time (Shima \& Schumacher, 2009). On the other hand, the kinematics and dynamics of UAVs are usually not considered, as opposed to the formulation of path/motion planning and TO problems.

\section{UAVRTOP taxonomy}

In this section, a taxonomy is proposed in order to help readers identify the key differences among various UAV routing problems and guide their research towards the development of new algorithms.

We have identified 20 attributes that are common to the UAV TO, PP, routing and TA literatures.

They define common features of UAVs routing problems, such as the kind of fleet, mission characteristics and flight dynamics. Attributes are further grouped into five classes. The first class collects the characteristics of UAVs, the second class represents the characteristics of waypoints, the third class describes the characteristics of the environment, the fourth class involves the characteristics of the launching point(s) and the last class is concerned about flight duration. These are listed in Table 1. The last two lines of this table are not part of the taxonomy, but they are important for understanding Appendix A.

Within class UAVs, the fleet and how the UAVs' kinematics and dynamics are modelled are considered as follows:

1. Multiple - A fleet of vehicles is available (as opposed to a single vehicle).

2. Heterogeneous - Heterogeneous fleet, i.e., the fleet is composed of vehicles with different characteristics (as opposed to a homogeneous fleet).

3. Fleet Size - The size of the fleet must be optimised (as opposed to a fixed fleet size).

4. Capacity - Vehicles are capacitated (as opposed to uncapacitated vehicles). A capacitated UAV might have, for example, a maximum flight endurance or maximum payload capacity.

5. Geometric - The vehicle's flight dynamics are neglected (as opposed to considering flight dynamics). For example, by using Euclidean distances between waypoints instead of flight distances.

6. Dubin's - Dubin's vehicles are used to model the UAVs.

7. EOM - A set of differential equations has been used to model the vehicles' kinematics and dynamics (as opposed to neglecting the dynamics).

The class Waypoints presents the attributes of the waypoints (vertices):

8. Multiple - Multiple waypoints must be visited (as opposed to a single waypoint or destination).

9. Unordered - The visiting order of waypoints is unknown (as opposed to a predefined order).

10. Visits - The waypoints can be visited multiple times (as opposed to a single visit for each waypoint). 
11. Constraints - Special mission constraints must be considered. For instance, time-windows, precedences, and special boundary conditions.

12. Covering Region - A continuous, but not necessarily convex, region (or airspace) is defined over the waypoint. We believe this characteristic is important to the UAVRTOP since most UAVs' sensors require at least a minimum radius of action in order to be effective.

The Environment class collects the attributes about the environment where the UAVs operate:

13. $3 D$ - The UAVs operate in a $3 \mathrm{D}$ space (as opposed to a $2 \mathrm{D}$ space).

14. Obstacles - The problem includes the presence of fixed or moving obstacles (as opposed to an obstacle-free environment).

15. Wind - The effects of wind are considered (as opposed to neglecting the wind effects).

16. Real-time - The problem must be solved in real-time. For example, waypoints and tasks arriving at random times and locations.

The class Launching groups the attributes about the number of launching points (depots):

17. Multiple - There are multiple launching points (as opposed to a single launching point).

18. Inter-depot - There are inter-depots available (e.g., for refuelling, battery replenishment or maintenance of the UAVs).

Papers are classified in class Time according to the way the flight time is considered in their models:

19. Fixed - The UAVs' flight times between arcs can be computed beforehand. This is a common characteristic of some PP methods (e.g., the Dubin's model).

20. Variable - The UAVs' velocities and flight times between arcs are optimisation variables.

In order to provide a survey of the most relevant and recent papers, we adopted the following procedure. Papers published since 2010 were collected from the following databases: The Web of Science, Google Scholar and ScienceDirect. We have limited our search to papers published in English. In order to cover the most common types of UAVs, we considered Unmanned Combat Aerial Vehicle (UCAV), Unmanned Aerial Systems (UAS) and aerial gliders in our search. The following keywords were used:

- UAV/UCAV/UAS/aerial glider trajectory optimisation

- $\mathrm{UAV} / \mathrm{UCAV} / \mathrm{UAS} /$ aerial glider PP

- UAV/UCAV/UAS/aerial glider motion planning

- UAV/UCAV/UAS/aerial glider task assignment

- UAV/UCAV/UAS/aerial glider routing

Papers that focus on Control Theory for UAVs were not reviewed. 
Table 1: Characteristics of the problems considered in this literature review.

\begin{tabular}{|c|c|c|}
\hline & UAVs & \\
\hline 1 & Multiple & A fleet of vehicles is available \\
\hline 2 & Heterogeneous & The fleet is heterogeneous \\
\hline 3 & Fleet size & The size of the fleet must be optimised \\
\hline 4 & Capacity & Vehicles are capacitated \\
\hline 5 & Geometric & The vehicle's flight dynamics are neglected \\
\hline 6 & Dubin's & A Dubin's vehicle model has been used \\
\hline \multirow[t]{2}{*}{7} & EOM & A set of EOMs is used to model the UAVs' flight dynamics \\
\hline & Waypoints & \\
\hline 8 & Multiple & Multiple waypoints must be visited \\
\hline 9 & Unordered & The visiting order of waypoints is unknown \\
\hline 10 & Visits & Waypoints can be visited multiple times \\
\hline 11 & Constraints & $\begin{array}{l}\text { Special mission constraints must be considered. (e.g., time-windows and boundary } \\
\text { conditions) }\end{array}$ \\
\hline \multirow[t]{2}{*}{12} & Covering Region & If there is a continuous covering region around the waypoints \\
\hline & Environment & \\
\hline 13 & $3 \mathrm{D}$ & The UAVs operate in a $3 \mathrm{D}$ space \\
\hline 14 & Obstacles & If obstacles are present \\
\hline 15 & Wind & The effects of wind are considered \\
\hline \multirow[t]{2}{*}{16} & Real-time & The problem must be solved in real-time \\
\hline & Launching (Depot) & \\
\hline 17 & Multiple & There are multiple launching points \\
\hline \multirow[t]{2}{*}{18} & Inter-depot & There are inter-depots available \\
\hline & Time & \\
\hline 19 & Fixed & The UAVs' flight times between arcs are known. \\
\hline \multirow[t]{3}{*}{20} & Variable & Flight times and velocities are optimisation variables \\
\hline & Approach & The type of algorithm used to solve the problem (Appendix A) \\
\hline & Application & A real-world motivation to solve the problem (Appendix A) \\
\hline
\end{tabular}




\section{Critical review of the recent literature}

balanced our analysis by considering articles dedicated to UAV TO/PP and UAV routing/TA. Papers devoted to technical and theoretical aspects of UAV flight dynamics were excluded from our analysis. Articles published in journals and conferences have been included in a number that we consider to be representative. Nonetheless, we apologise for any inadvertent omission of relevant papers.

The selected papers have been organised into Table 2. Each line of this table corresponds to one article and the meaning of each column relates to the numbering in Table 1. Each time an attribute is present in a paper the respective column is marked with "X". Therefore, an empty cell indicates that its corresponding paper has not addressed the attribute indicated by this cell's column. A table with a detailed description of methods and applications for each article can be found in the Appendix A. Statistics about Table 2 are provided in Table 3.

Three types of articles can be identified in Table 2. Papers focusing on UAV routing and TA can be identified by the presence of attributes 8 and 9 . The second type, which involves papers on UAV PP and TO, exclusively, correspond to the ones where attribute 9 is absent. The third type consists of articles that integrate UAV routing and PP or UAV routing and TO. The former can be identified by the presence of attributes 5 or 6 together with 8 and 9 , while the latter can be identified by the presence of attributes 7,8 and 9 together.

In Table 2 it can be seen that $70 \%$ of the articles considered a fixed flight time. This indicates that most of the UAV literature is concerned with routing and PP algorithms, where constant velocity along the trajectories is a common assumption. The EOMs of the vehicles were employed in $17.1 \%$ of the articles. In $53.8 \%$ of the papers on PP that applied a Dubin's model (which consist of only $18.6 \%$ of the total number of papers), the flight time has been considered as a variable.

Multiple UAVs were considered in $25 \%$ of the papers dealing with TO and PP. An interesting fact arises counting the number of papers dealing with multiple UAVs and their EOMs. There seems to be a preference for using PP methods and the Dubin's model when a fleet of UAVs is taken into account. One can notice that the preferred strategy is to simplify the physical models of the UAVs so as to make the problem of designing multiple flyable routes more tractable. This happens in $44.4 \%$ of the articles on UAV PP and TO and in all the articles on UAV routing and TA.

Around $37.5 \%$ of the papers on TO and PP problems dealt with visiting multiple waypoints. However, only $14.3 \%$ attempted to integrate PP and TO to routing decisions. Among them, three papers employed the UAVs' EOMs. This gives an indication that integrated routing and TO is yet to be fully investigated in the literature.

Regarding environmental conditions, $40 \%$ of the papers have studied three dimensional problems. Obstacle avoidance was tackled in $22.8 \%$ of the articles. Only a few studies (10\%) included the effects of the wind in the UAVs' trajectories. In addition, only $7.1 \%$ of the papers studied real-time applications.

In $78.5 \%$ of the papers articles focusing on UAV routing and TA, a fleet of UAVs was considered. A large amount $(85.7 \%)$ of the articles on routing and TA have either neglected or simplified the dynamics of the UAVs. Approximately $18 \%$ of the articles have modelled the UAVs as Dubin's vehicles. There is 
some overlap between these papers since some of them employ more than one methodology. This suggests the preference for simplified vehicle models when dealing with UAV routing.

Table 2: Summary of the taxonomic review on 70 selected papers.

\begin{tabular}{|c|c|c|c|c|c|c|c|c|c|c|}
\hline Author(s) & $\frac{\mathrm{U}}{12}$ & $\frac{\text { UAVs }}{3456}$ & $\frac{\text { Wayr }}{8910}$ & ooint & & $\frac{\text { En }}{314}$ & & $\frac{\text { Dep. }}{1718}$ & & $\frac{\text { ime }}{220}$ \\
\hline Al-Sabban et al. (2012) & & & & & & & & & & \\
\hline Babel (2011) & & $\times$ & & & & $\times$ & & & $x$ & \\
\hline Babel (2012) & & $x \times$ & & & $x$ & & & & & $\times$ \\
\hline Bae et al. (2015) & & $x \times$ & $x$ & & & & & & $x$ & \\
\hline Baiocchi (2014) & & $x$ & $x$ & & $x$ & & & & $x$ & \\
\hline Bandeira et al. (2015) & & $x$ & $x \times$ & $x$ & & & & & $x$ & \\
\hline Bednowitz et al. ( 20 & $x$ & $x$ & $x \times$ & $x$ & & & & & $x$ & \\
\hline Besada-Portas et al. (2010) & $x$ & $x$ & $\times$ & & $x$ & & & & $x$ & \\
\hline Besada-Portas et al. & $x$ & $\times$ & $\times$ & & $x$ & & & & $x$ & \\
\hline Casbeer \& Holsapple & $x \times$ & $x \times$ & $x \times$ & $x$ & & & & $x$ & $x$ & \\
\hline Chakrabarty \& Langelaan (2011) & & $x$ & $\times$ & & $x$ & & $x$ & & & $x$ \\
\hline al. $(2016)$ & $x$ & & $x \times$ & $\times$ & $x$ & $x$ & & & & $x$ \\
\hline Choe et al. (2016) & $x$ & $\times$ & $\times$ & & $x$ & & & $x$ & $x$ & \\
\hline al. (2013) & $x$ & $x$ & $x \times$ & $x$ & $x$ & & & $x$ & $x$ & \\
\hline Cons et al. (2014) & & $x$ & $x \times$ & & & & & & $x$ & \\
\hline Crispin (2016) & $x$ & & $x$ & & $x$ & & & & $x$ & \\
\hline Dilão \& Fonseca (2013) & & & & & $x$ & & & & & $x$ \\
\hline Edison \& Shima (2011) & $x \times$ & $x$ & $x \times$ & & & & & & $x$ & \\
\hline Enri & $x$ & $x \times$ & $x \times$ & $\times \times$ & & & & $x$ & $x$ & \\
\hline Evers et ? & & $x \times$ & $x \times$ & $\times$ & & & $x$ & & $x$ & \\
\hline Fai & $x$ & $x$ & $x \times$ & $x$ & & & & $x$ & $x$ & \\
\hline Filippis et al. (2011) & & $x$ & & & & $x$ & & & $x$ & \\
\hline Forsmo (2012) & $x$ & & $<\times \times$ & & & & & $x$ & $x$ & \\
\hline h \& Müllenstedt (2015) & $x \times>$ & $x \times$ & $x \times x$ & $\times \times$ & $x$ & & $\times$ & $x$ & & $x$ \\
\hline Furini et al. (2016) & & $\times$ & $\times \times$ & $x$ & & $x$ & & & $x$ & \\
\hline Gottlieb \& Shi & $x$ & $x$ & $\times \times$ & $\times$ & & $\times$ & & $x$ & & $x$ \\
\hline Gue & $x$ & $\times \times \times$ & $x \times$ & $x$ & & & & $x$ & $\times$ & \\
\hline Han & & $x \times$ & & & & $x$ & & & $x$ & \\
\hline Her & & $x$ & $x \times$ & $\times \times$ & & $x$ & $\times \times$ & & & $x$ \\
\hline & $x$ & $x$ & $x$ & & & $x$ & & & $\times$ & \\
\hline $\mathrm{Hu}$ & $x$ & $x \times$ & $x \times$ & & & & & $x$ & $x$ & \\
\hline Jais & & $\times$ & & $x$ & $x$ & $x$ & & & $x$ & \\
\hline Jian & $x$ & $x$ & $\times \times$ & $x$ & & & & & $x$ & \\
\hline Kaq & & $x$ & $x$ & & $x$ & & & & $x$ & \\
\hline al. (2016) & $x$ & $x$ & $x \times$ & & & & & $x$ & $x$ & \\
\hline & & & $x \times$ & & & & & & & $x$ \\
\hline & $x \times$ & $\times$ & $\times \times \times$ & $\times$ & $x$ & & & & $\times$ & \\
\hline & $x \times$ & $x \ddot{x}$ & $x \times x$ & & & & & $\times \times$ & $x$ & \\
\hline & & $\times$ & & & & $\times$ & & & & $x$ \\
\hline Liu & & $x$ & $\times$ & & $x$ & & & & $x$ & \\
\hline Man & $x$ & $\times$ & $\times \times$ & $x$ & & & & $x$ & & $x$ \\
\hline & $x$ & $x \times$ & $\times \times \times$ & $x$ & & & & $\times \times$ & $x$ & \\
\hline & $x$ & $x \times$ & $x \times$ & & & & & & $x$ & \\
\hline $\mathrm{Mu}$ & $x \times$ & $x \times$ & $x \times$ & $x$ & & & & $\times$ & $x$ & \\
\hline & $\times \times$ & $x \times$ & $x \times \times$ & & & & & $\times$ & $x$ & \\
\hline & & $x$ & $\times \times$ & & & $x$ & $\times$ & $\times$ & & $x$ \\
\hline & & $\times$ & $\times \times \times$ & $\times$ & $x$ & & & & & $x$ \\
\hline & $x \times$ & $x$ & $x \times$ & $\times$ & $x$ & & & & $\times$ & \\
\hline Park & & $x \times$ & $x \times \times$ & $x$ & & & & & $x$ & \\
\hline Pepy & & & $x$ & & & & & & & $\times$ \\
\hline Pha & & $x$ & & & $\times$ & & & & $x$ & \\
\hline Rog & & & & & & & & & & $\times$ \\
\hline Sha & & $x$ & & & & & & & $x$ & \\
\hline & & & & & $x$ & & $x$ & & & $x$ \\
\hline & $x \times$ & $x \times$ & $\times$ & $x$ & & & & $\times \times$ & $\times$ & \\
\hline Stu & $x$ & $x \times$ & $\times \times \times$ & $x$ & & & & $\times$ & $x$ & \\
\hline & & $\times \times$ & $\times \times$ & $x$ & $x$ & & & $\times$ & $x \times$ & \\
\hline & & & & & & & $x$ & & & $x$ \\
\hline & $x$ & $x \times$ & $\times \times$ & & & & & & $x$ & \\
\hline & $x$ & $\times$ & $x \times$ & $x$ & & $\times$ & & & $x$ & \\
\hline War & $\times \times$ & $x \times$ & $x \times$ & $x$ & $x$ & $x$ & & $x$ & $x$ & \\
\hline & & $\times$ & & & $x$ & & & & $x$ & \\
\hline Wu et al. (2011) & & $x$ & & $x$ & $x$ & $x$ & & & $x$ & \\
\hline Xu et al. (2017) & $x$ & & & $x$ & $x$ & $x$ & & & & $x$ \\
\hline Yakı & $x$ & $x \times$ & $\times x$ & & & & & & $x$ & \\
\hline Yan & $x$ & $\times \times$ & $\times \times \times$ & & $x$ & & & $x$ & $x$ & \\
\hline et al. (2016) & & $x$ & & $x$ & $x$ & & & & & $x$ \\
\hline$(2011)$ & & $x$ & & $x$ & $x$ & $x$ & $\times$ & & $\times$ & \\
\hline Zha & & & $<\times x$ & & $x$ & & $\times \times$ & & & $x$ \\
\hline Zhang et al. (2014) & $x$ & $x$ & $x \times$ & $\times \times$ & & & & $\times$ & & $x$ \\
\hline
\end{tabular}

Table 3 illustrates other differences between the literature on UAV TO/PP and UAV routing/TA. Each row of Table 3 shows four classes that were defined in the proposed taxonomy and their respective frequencies (defined as the number of non-empty cells divided by the total number of cells in that class). For example, for the articles tackling TO and PP, the number of non-empty cells for class Depot is 3 and the total number of cells for the same class is 64 . Hence the density of class Depot for TO/PP papers is $3 / 64=0.047$. One can notice that while the routing/TA literature is able to include more VRP-like attributes (like multiple UAVs and waypoints), the literature on TO/PP is more concerned 
about modelling environmental aspects. Including environmental attributes (such as obstacles and wind) is usually possible when the UAVs physical models are integrated to the optimisation problem.

Table 3: Densities per class for each classification.

\begin{tabular}{lcc}
\hline Class & TO/PP & routing/TA \\
\hline UAVs & $20.1 \%$ & $37.8 \%$ \\
Waypoints & $11.9 \%$ & $61.4 \%$ \\
Environment & $26.6 \%$ & $9.8 \%$ \\
Depot & $4.7 \%$ & $30.4 \%$ \\
\hline
\end{tabular}

In addition, the number of articles using a fixed flight time between waypoints is higher in the are very popular among articles considering UAV routing and TA. Being exact methods more popular in the UAV routing papers (Figure 3c) and heuristics and metaheuristics more popular in the UAV TA 
articles (Figure 3d).

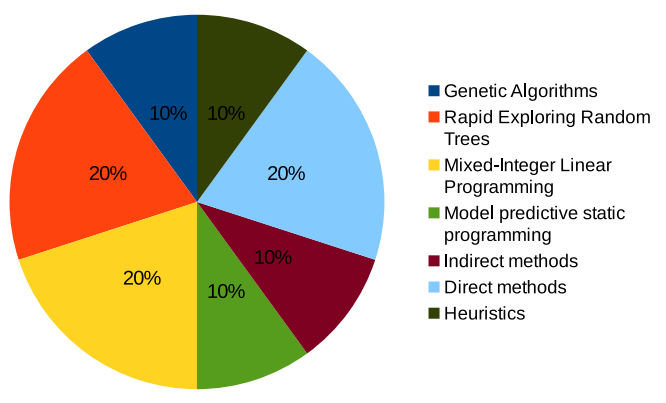

(a) Articles on UAV trajectory optimisation.

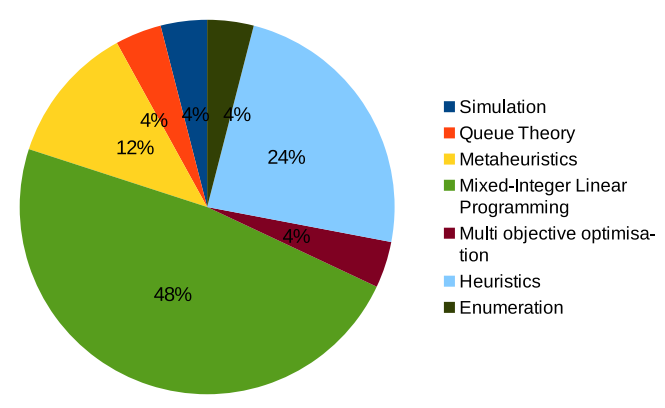

(c) Articles on UAV routing.

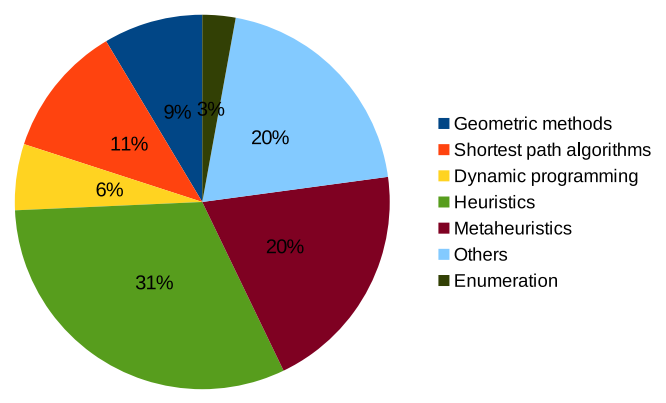

(b) Articles on UAV path planning.

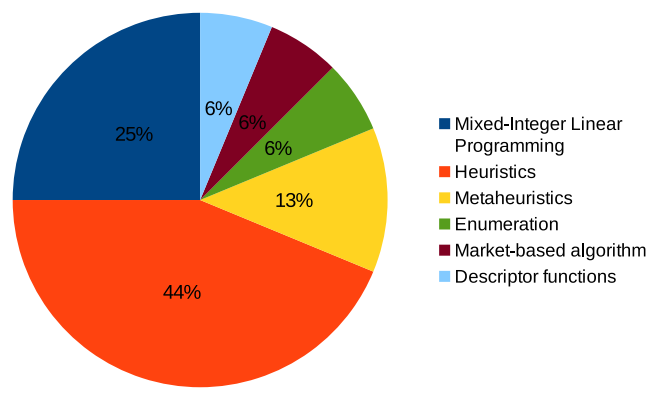

(d) Articles on UAV task assignment.

Figure 3: Overview of methods and algorithms employed in the 70 selected papers.

\subsection{Integrating routing and trajectory optimisation}

Hereafter we highlight the contribution of articles that studied UAV routing and TO in an integrated framework. Such articles can be identified in Table 2 by attributes 7, 8 and 9 being marked with "X". These papers present alternative frameworks to the UAVRTOP formulation presented in Section 2.

Zhang et al. (2012) investigated the problem of routing a combat UCAV in a 3D environment through stationary ground targets whilst avoiding no-fly threat areas. In order for the attacks to succeed, the UCAV must fly within the targets' allowable attack region (which consists of a hollow-cone-like airspace around the target) and respect projectile release attitude and velocity constraints. The UCAV was modelled by high fidelity 3DOF EOMs taking wind velocities into account.

In order to solve this problem, Zhang et al. (2012) propose a hierarchical heuristic with two levels. In the first level, the vehicle's state space is discretised into a set of feasible points that intersects the targets' allowable attack region through the use of a modified probabilistic road map method. Then, for every pair of sampled points not in the same target a TO problem was solved to obtain feasible trajectories (with respect to the vehicle's dynamics and operational constraints) and their respective costs. The second decision level consists of solving a Generalised Travelling Salesman Problem (GTSP) over the network produced in the first level. This is accomplished by transforming the GTSP into an instance of the Asymmetric TSP by means of the noon-bean transformation method. The Lin-Kernighan heuristic was then employed to solve the ATSP. In addition, the authors embedded this algorithm into a real-time framework in order to make this approach more flexible for practical applications. Numerical 
experiments showed that this approach is computationally intensive. The authors reported that roughly 50 minutes were necessary to solve a test case with three targets and one no-fly zone.

Fügenschuh \& Müllenstedt (2015) studied the problem of designing and routing a fleet of heterogeneous UAVs over a set of waypoints. The waypoints have to be selected from a list where a score was associated to each waypoint. The objective was to maximise the total score (defined as the sum of the individual scores) whilst minimising the total flight time. The UAVs' motion was modelled by piecewise linear dynamics based on Newton's laws of motion. The advantage of using this model lies on its simplicity, since the discretised version of these EOMs is also linear. On the other hand, the accuracy of such a model regarding UAVs flight dynamics is limited. In order to represent the range of the UAVs' sensors, the waypoints were considered to rest inside a sphere. A waypoint would be considered visited if a UAV passes through its covering sphere. No-fly zones and collision avoidance among the UAVs were also considered. Finally, different locations could be chosen to launch each UAV.

The authors proposed a Mixed-Integer Non-linear Programming (MINLP) formulation to this problem, which was linearised and could be solved by a commercial MILP optimisation software. Eight instances were created by varying the number of waypoints between 3-15, the number of no-fly zones between 0-3 and the number of UAVs between 1-2. Computational experiments showed that bigger instances with 10-15 waypoints could not be solved within one hour. The computation time required to solve smaller problems to optimality varied between 57-3400 seconds.

A similar approach was presented by Forsmo (2012). The author applied Newton's second law in order to model the motion of the UAVs. However, constraints on the magnitudes of forces, velocities and yaw rates were also imposed, which increased the complexity of the physical representation of the UAVs. Several operational constraints were considered, such as obstacles and collision avoidance. Scenarios with up to two UAVs and multiple waypoints were generated. A MILP formulation was proposed in order to find minimum flight time trajectories visiting all waypoints subject to mission and operational constraints. Computational experiments were performed over 5 test cases, constructed by varying the number of UAVs (1 or 2), waypoints (6 or 8) and by imposing, or not imposing, a visiting order. The authors showed that CPU times could be reduced by decreasing the flight time horizon.

\section{Conclusions and directions for future research}

The UAVRTOP is a routing problem that takes into account the flight dynamics of UAVs. UAV routing problems usually ignore flight dynamics, while work on UAV trajectory optimisation usually ignores any routing aspects. Coupling these two important aspects leads to a more realistic approach that allows the design of optimal routes and trajectories for a fleet of UAVs flying simultaneously.

This problem arises from the current development of UAV technology and the vast number of applications that these vehicles can be used for. In this paper, we first formalised the UAVRTOP. Next, an introduction to TOPs, VRPs and their variants has been provided. In addition, we introduced a taxonomy capable of classifying UAV routing/TA and UAV TO/PP problems according to their most relevant features. This taxonomy included 20 common attributes from the literature. Finally, we applied the proposed taxonomy to 70 recent papers. 
The literature on UAVs routing problems has been surveyed and a lack of articles integrating UAV routing and TO has been identified. In particular, the UAVs' flight dynamics is often simplified or neglected. In many cases the behaviour of UAVs cannot be satisfactorily approximated only by their kinematics, as in the case of terrestrial robots (Forsmo, 2012). We believe that integrating the UAVs' system dynamics into routing problems is a key concept for complex operations. A realistic routing and TO algorithm must take into account the vehicle's kinematics and dynamics. In addition, by considering the UAVs' EOMs one can also better approximate, for example, the vehicles' energy consumption, which is highly important for UAVs with limited battery duration. Modelling energy consumption is an issue that needs further investigation in the UAV routing literature.

Flight safety is an important aspect in connection with the use of UAVs. Most research on UAV routing does not consider, for example, collision avoidance and wind conditions. This is important, e.g., for goods distribution within urban areas where collisions with buildings and manned aircraft must be avoided and the fleet of UAVs must operate in a robust and reliable way.

Usually, research on UAV path and route planning concentrates on modelling kinematics. In many articles about UAV routing and TA even the kinematics are neglected. Models taking into account the forces acting on these vehicles, the interaction with wind and their manoeuvring capabilities could possibly result in computationally expensive formulations, but such models might allow for more realistic solutions.

Mathematical formulations and algorithms capable of tackling complex unmanned aerial systems in a routing framework have recently appeared in the literature. A first step in this direction has been made by Zhang et al. (2012), Forsmo (2012) and Fügenschuh \& Müllenstedt (2015). Zhang et al. (2012) proposed a heuristic method based on the 3DOF EOMs of a UAV. Whereas Forsmo (2012) and Fügenschuh \& Müllenstedt (2015) developed MILP formulations based on simplified dynamic equations. Only problems with limited size have been solved by the aforementioned authors. Therefore, the development of efficient frameworks for solving UAVRTOPs still raises challenging research questions that need to be answered.

\section{Appendix A. Methods and applications for the selected literature}

Table A.4 highlights the methods and practical applications addressed in the 70 selected papers. 
Table A.4: Summary of methods and applications on 70 selected papers.

\begin{tabular}{|c|c|c|}
\hline Authors & Approach & Application \\
\hline Al-Sabban et al. (2012) & Markov decision process & Path planning in uncertain wind conditions \\
\hline Babel (2011) & Shortest path algorithms & UAV path planning with obstacles \\
\hline Babel (2012) & Shortest path algorithms & Path planning in a risk environment \\
\hline Bae et al. (2015) & Dynamic programming and heuristics & Risk-constrained shortest path for UCAV \\
\hline Baiocchi (2014) & Heuristic algorithms & Path planning for aerial photography \\
\hline Bandeira et al. (2015) & Heuristic algorithms & UAV routing for aerial photography \\
\hline Bednowitz et al. (2012) & Simulation model & UAV routing in dynamic environment \\
\hline Besada-Portas et al. (2010) & Evolutionary algorithms & Real-time UAV path planning \\
\hline Besada-Portas et al. (2013) & Evolutionary algorithms & Real-time UAV path planning \\
\hline Casbeer \& Holsapple (2011) & Column generation & UAV TA with precedence \\
\hline Chakrabarty \& Langelaan (2011) & Energy map method & Path planning for soaring UAVs \\
\hline Chen et al. (2016) & Genetic algorithm & Multi UAV trajectory optimisation \\
\hline Choe et al. (2016) & Pythagorean hodograph bézier curves & Cooperative path planning \\
\hline Cobano et al. (2013) & Rapid exploring random trees & Cooperative trajectory optimisation \\
\hline Cons et al. (2014) & Heuristic algorithms & Integrated TA and path planning \\
\hline Crispin (2016) & Rapid exploring random trees & Path planning for aerial gliders \\
\hline Dilão \& Fonseca (2013) & Heuristic algorithms & Path planning for a hypersonic glider \\
\hline Edison \& Shima (2011) & Genetic algorithm & Integrated TA and path planning \\
\hline Enright et al. (2015) & Queueing theory & UAV routing in stochastic environments \\
\hline Evers et al. (2014) & ILS metaheuristic & UAV orienteering problem with time windows \\
\hline Faied et al. (2010) & Mixed-Integer Linear Programming & Multi UAV routing problem \\
\hline Filippis et al. (2011) & Shortest path algorithms & UAV path planning with obstacles \\
\hline Forsmo $(2012)$ & Mixed-Integer Linear Programming & UAV routing and trajectory optimisation \\
\hline Fügenschuh \& Müllenstedt (2015) & Mixed-Integer Linear Programming & UAV routing and trajectory optimisation \\
\hline Furini et al. (2016) & Mixed-Integer Linear Programming & Time dependent UAV routing problem \\
\hline Gottlieb \& Shima (2015) & Enumerative and heuristic algorithms & Integrated TA and path planning \\
\hline Guerriero et al. (2014) & Multi-objective optimisation & UAV routing with time windows \\
\hline Han et al. (2014) & Dynamic programming & UAV path planning \\
\hline Henchey et al. (2016) & Enumerative and heuristic algorithms & UAV routing problems \\
\hline Huang et al. (2016) & Ant colony optimisation & Multi UAV path planning \\
\hline Hu et al. (2015b) & Ant colony optimisation & UAV task assignment \\
\hline Jaishankar \& Pralhad (2011) & Multi criteria decision analysis & UAV path planning \\
\hline Jiang \& Ng (2011) & Mixed-Integer Linear Programming & Multi UAV routing problem \\
\hline Kagabo (2010) & Fuzzy Logic & Path planning for aerial gliders \\
\hline Kivelevitch et al. (2016) & Market-based algorithm & UAVs TA problem \\
\hline Kumar \& Padhi (2013) & Model predictive static programming & UAV trajectory optimisation \\
\hline
\end{tabular}


Table A.4 - continued from previous page

\begin{tabular}{|c|c|c|}
\hline Authors & Approach & Application \\
\hline Kwak et al. (2013) & Heuristic algorithms & Generalised UAVs TA \\
\hline Levy et al. (2014) & Heuristic algorithms & UAVs routing with refuelling depots \\
\hline Liu et al. (2013) & Heuristic algorithms & Real-time UAV path planning \\
\hline Liu et al. (2016) & Collocation interval analysis method & UAV path planning \\
\hline Manyam et al. (2015) & Lagrangian Relaxation & Multi depot UAVs routing \\
\hline Mersheeva (2015) & Heuristics and constraint programming & UAV routing in disaster assessment \\
\hline Mufalli et al. (2012) & Mixed-Integer Linear Programming and heuristics & UAVs routing problems \\
\hline Murray \& Karwan (2010) & Mixed-Integer Linear Programming & UAVs dynamic TA and routing \\
\hline Murray \& Karwan (2013) & Branch-and-bound & UAVs dynamic routing \\
\hline Myers et al. (2016) & Shortest path algorithm & Real-time UAV path planning \\
\hline Nguyen et al. (2015) & Look-up tables & Path planning for aerial gliders \\
\hline Niccolini et al. (2010) & Descriptor functions methodology & Multi UAV TA problem \\
\hline Park et al. (2012) & Heuristic algorithms & UAV routing \\
\hline Pepy \& Hérissé (2014) & Indirect shooting method & Trajectory optimisation for an aerial glider \\
\hline Pharpatara et al. (2015) & Geometric path planning & Path planning for a hypersonic glider \\
\hline Rogowski \& Maroński (2011) & Direct pseudospectral method & Trajectory optimisation for an aerial glider \\
\hline Shanmugavel (2013) & Bayesian rule-based algorithm & UAV path planning \\
\hline Silva et al. (2015) & Non-linear programming & Trajectory optimisation for an aerial glider \\
\hline Song et al. (2016) & Mixed-Integer Linear Programming and heuristics & Multi UAV TA problem \\
\hline Stump \& Michael (2011) & Mixed-Integer Linear Programming & Multi UAV routing problem \\
\hline Sundar \& Rathinam (2014) & Heuristic and approximation algorithms & UAV routing with refueling depots \\
\hline Techy et al. (2010) & Heuristic algorithm & UAV path planning in uniform wind \\
\hline Thi et al. (2012) & Exact and heuristic algorithms & UAVs task assignment \\
\hline Vilar \& Shin (2013) & Heuristic algorithm & Communication-aware TA problem \\
\hline Wang et al. (2015) & Heuristic algorithms & Multi UAV TA problem \\
\hline Wang et al. (2016) & Population-based algorithms & UAV path planning \\
\hline Wu et al. (2011) & Genetic Algorithm & UAV path planning \\
\hline Xu et al. (2017) & Gradient-descent algorithm & UAV path planning \\
\hline Yakıcı $(2016)$ & Ant Colony Optimisation & UAV location and routing problem \\
\hline Yang et al. (2015) & Heuristic algorithms & UAV path planning \\
\hline Yomchinda et al. (2016) & Parametrization techniques and heuristics & Aircraft path planning \\
\hline Zhang et al. (2011) & Differential Evolution Algorithm & UAVs real-time path planning \\
\hline Zhang et al. (2012) & Heuristic algorithm & UAV routing and trajectory optimisation \\
\hline Zhang et al. (2014) & Memetic Algorithm & UAV routing problem \\
\hline
\end{tabular}


Al-Sabban, W. H., Gonzalez, L. F., Smith, R. N., \& Wyeth, G. F. (2012). Wind-energy based path planning for electric unmanned aerial vehicles using Markov Decision Processes. In Proceedings of the IEEE/RSJ International Conference on Intelligent Robots and Systems (pp. 1-6). Algarve: IEEE. doi:10.1109/ICRA.2013.6630662.

Alidaee, B., Gao, H., \& Wang, H. (2010). A note on task assignment of several problems. Computers $\&$ Industrial Engineering, 59, 1015-1018. doi:10.1016/j.cie.2010.07.010.

Alidaee, B., Wang, H., \& Landram, F. (2011). On the Flexible Demand Assignment Problems: Case of Unmanned Aerial Vehicles. IEEE Transactions on Automation Science and Engineering, 8, 865-868. doi:10.1109/TASE.2011.2159709.

Alighanbari, M., \& How, J. P. (2008). A robust approach to the UAV task assignment problem. International Journal of Robust and Nonlinear Control, 18, 118-134. doi:10.1002/rnc.1268.

Babel, L. (2011). Trajectory planning for unmanned aerial vehicles: a network optimization approach. Mathematical Methods of Operations Research, 74, 343-360. doi:10.1007/s00186-011-0366-1.

Babel, L. (2012). Three-dimensional Route Planning for Unmanned Aerial Vehicles in a Risk Environment. Journal of Intelligent \& Robotic Systems, 71, 255-269. doi:10.1007/s10846-012-9773-7.

Bae, K.-Y., Kim, Y.-D., \& Han, J.-H. (2015). Finding a risk-constrained shortest path for an unmanned combat vehicle. Computers \& Industrial Engineering, 80, 245-253. doi:10.1016/j.cie.2014.12.016.

Baiocchi, V. (2014). Development of a software to optimize and plan the acquisitions from UAV and a first application in a post-seismic environment. European Journal of Remote Sensing, 47, 477-496. doi:10.5721/EuJRS20144727.

Bandeira, T. W., Coutinho, W. P., Brito, A. V., \& Subramanian, A. (2015). Analysis of Path Planning Algorithms Based on Travelling Salesman Problem Embedded in UAVs. In 2015 Brazilian Symposium on Computing Systems Engineering (SBESC) (pp. 70-75). doi:10.1109/SBESC.2015.20.

Barraquand, J., \& Latombe, J.-C. (1991). Robot Motion Planning: A Distributed Representation Approach. The International Journal of Robotics Research, 10, 628-649. doi:10.1177/ 027836499101000604.

Beard, R. W., \& McLain, T. W. (2012). Small Unmanned Aircraft: Theory and Practice. Princeton University Press.

Bednowitz, N., Batta, R., \& Nagi, R. (2012). Dispatching and loitering policies for unmanned aerial vehicles under dynamically arriving multiple priority targets. Journal of Simulation, 8, 9-24. doi:10.1057/jos.2011.22.

Bektas, T. (2006). The multiple traveling salesman problem: an overview of formulations and solution procedures. Omega, 34, 209-219. doi:10.1016/j.omega.2004.10.004. 
Bertsekas (1979). Stochastic Optimal Control: The Discrete Time Case. Elsevier Science.

Besada-Portas, E., de la Torre, L., Moreno, A., \& Risco-Martín, J. L. (2013). On the performance comparison of multi-objective evolutionary UAV path planners. Information Sciences, 238, 111-125. doi:10.1016/j.ins.2013.02.022.

Besada-Portas, E., Torre, L. d. 1., Cruz, J. M. d. 1., \& Andrés-Toro, B. d. (2010). Evolutionary Trajectory Planner for Multiple UAVs in Realistic Scenarios. IEEE Transactions on Robotics, 26, 619-634. doi:10.1109/TR0.2010.2048610.

Betts, J. T. (1998). Survey of Numerical Methods for Trajectory Optimization. Journal of Guidance, Control, and Dynamics, 21, 193-207. doi:10.2514/2.4231.

Betts, J. T. (2001). Practical methods for optimal control using nonlinear programming. Advances in design and control. Philadelphia, PA: Society for Industrial and Applied Mathematics.

Braekers, K., Ramaekers, K., \& Van Nieuwenhuyse, I. (2016). The vehicle routing problem: State of the art classification and review. Computers \& Industrial Engineering, 99, 300-313. doi:10.1016/ j.cie.2015.12.007.

Bravo, R., \& Leiras, A. (2015). Literature Review of the Applications of UAVs in Humanitairan Relief. In Perspectivas Globais para a Engenharia de Produção (pp. 1-15). Fortaleza-CE, Brazil.

Bryson, A. E. (1975). Applied Optimal Control: Optimization, Estimation and Control. CRC Press.

Bryson, A. E. (2002). Applied Linear Optimal Control Paperback with CD-ROM: Examples and Algorithms. Cambridge University Press.

Casbeer, D. W., \& Holsapple, R. W. (2011). Column generation for a UAV assignment problem with precedence constraints. International Journal of Robust and Nonlinear Control, 21, 1421-1433. doi:10.1002/rnc.1722.

Chakrabarty, A., \& Langelaan, J. W. (2011). Energy-Based Long-Range Path Planning for SoaringCapable Unmanned Aerial Vehicles. Journal of Guidance, Control, and Dynamics, 34, 1002-1015.

Chen, Y., Yu, J., Mei, Y., Zhang, S., Ai, X., \& Jia, Z. (2016). Trajectory optimization of multiple quad-rotor UAVs in collaborative assembling task. Chinese Journal of Aeronautics, 29, 184-201. doi:10.1016/j.cja.2015.12.008.

Choe, R., Puig-Navarro, J., Cichella, V., Xargay, E., \& Hovakimyan, N. (2016). Cooperative Trajectory Generation Using Pythagorean Hodograph Bézier Curves. Journal of Guidance, Control, and Dynamics, 39, 1-20. doi:10.2514/1.G001531.

Cobano, J. A., Alejo, D., Sukkarieh, S., Heredia, G., \& Ollero, A. (2013). Thermal detection and generation of collision-free trajectories for cooperative soaring UAVs. In 2013 IEEE/RSJ International Conference on Intelligent Robots and Systems (pp. 2948-2954). doi:10.1109/IROS.2013.6696774. 
Colasurdo, G., Zavoli, A., Longo, A., Casalino, L., \& Simeoni, F. (2014). Tour of Jupiter Galilean moons: Winning solution of GTOC6. Acta Astronautica, 102, 190-199. doi:10.1016/j.actaastro.2014.06.003.

Eksioglu, B., Vural, A. V., \& Reisman, A. (2009). The vehicle routing problem: A taxonomic review. Computers E Industrial Engineering, 57, 1472-1483. doi:10.1016/j.cie.2009.05.009.

Elston, J., Argrow, B., Stachura, M., Weibel, D., Lawrence, D., \& Pope, D. (2014). Overview of Small Cons, M. S., Shima, T., \& Domshlak, C. (2014). Integrating Task and Motion Planning for Unmanned Aerial Vehicles. Unmanned Systems, 02, 19-38. doi:10.1142/S2301385014500022.

Conway, B. A. (2010). Spacecraft Trajectory Optimization. Cambridge University Press.

Cordeau, J.-F., Laporte, G., Savelsbergh, M. W. P., \& Vigo, D. (2007). Chapter 6 Vehicle Routing. In Handbooks in Operations Research and Management Science (pp. 367-428). Elsevier volume 14 of Transportation. doi:10.1016/S0927-0507(06)14006-2.

Crispin, C. (2016). Path Planning Algorithms for Atmospheric Science Applications of Autonomous Aircraft Systems. Ph.D. Thesis University of Southampton, Southampton, UK.

Darrah, M., Fuller, E., Munasinghe, T., Duling, K., Gautam, M., \& Wathen, M. (2012). Using Genetic Algorithms for Tasking Teams of Raven UAVs. Journal of Intelligent $\& 3$ Robotic Systems, 70, 361-371. doi:10.1007/s10846-012-9696-3.

Delahaye, D., Puechmorel, S., Tsiotras, P., \& Feron, E. (2014). Mathematical Models for Aircraft Trajectory Design: A Survey. In E. N. R. Institute (Ed.), Air Traffic Management and Systems number 290 in Lecture Notes in Electrical Engineering (pp. 205-247). Springer Japan. doi:10.1007/ 978-4-431-54475-3_12.

Dilão, R., \& Fonseca, J. (2013). Dynamic Trajectory Control of Gliders. In Q. Chu, B. Mulder, D. Choukroun, E.-J. v. Kampen, C. d. Visser, \& G. Looye (Eds.), Advances in Aerospace Guidance, Navigation and Control (pp. 373-386). Springer Berlin Heidelberg. doi:10.1007/978-3-642-38253$6 \backslash 23$.

Edison, E., \& Shima, T. (2011). Integrated task assignment and path optimization for cooperating uninhabited aerial vehicles using genetic algorithms. Computers $\mathcal{E}$ Operations Research, 38, 340-356. doi:10.1016/j.cor.2010.06.001.

Fixed-Wing Unmanned Aircraft for Meteorological Sampling. Journal of Atmospheric and Oceanic Technology, 32, 97-115. doi:10.1175/JTECH-D-13-00236.1.

Enright, J. J., Frazzoli, E., Pavone, M., \& Savla, K. (2015). UAV Routing and Coordination in Stochastic, Dynamic Environments. In K. P. Valavanis, \& G. J. Vachtsevanos (Eds.), Handbook of Unmanned Aerial Vehicles (pp. 2079-2109). Springer Netherlands.

605 Evers, L., Barros, A. I., Monsuur, H., \& Wagelmans, A. (2014). Online stochastic UAV mission planning with time windows and time-sensitive targets. European Journal of Operational Research, 238, 348362. doi:10.1016/j.ejor.2014.03.014. 
Faied, M., Mostafa, \& Girard, A. (2010). Vehicle Routing Problem Instances: Application to Multi-UAV Mission Planning. In AIAA Guidance, Navigation, and Control Conference Guidance, Navigation, and Control and Co-located Conferences (pp. 1-11). American Institute of Aeronautics and Astronautics.

Filippis, L. D., Guglieri, G., \& Quagliotti, F. (2011). Path Planning Strategies for UAVS in 3d Environments. Journal of Intelligent \& Robotic Systems, 65, 247-264. doi:10.1007/s10846-011-9568-2.

Fisch, F. (2011). Development of a Framework for the Solution of High-Fidelity Trajectory Optimization Problems and Bilevel Optimal Control Problems. Ph.D. Thesis Technical University of Munich Munich, Germany.

Forsmo, E. J. (2012). Optimal Path Planning for Unmanned Aerial Systems. Msc. Thesis Norwegian University of Science and Technology, Norway.

Fügenschuh, A., \& Müllenstedt, D. (2015). Flight Planning for Unmanned Aerial Vehicles. Technical Report AMOS \#34(2015) Helmut Schmidt University / University of the Federal Armed Forces Hamburg.

Furini, F., Persiani, C. A., \& Toth, P. (2016). The Time Dependent Traveling Salesman Planning Problem in Controlled Airspace. Transportation Research Part B: Methodological, 90, 38-55. doi:10.1016/ j.trb.2016.04.009.

Galceran, E., \& Carreras, M. (2013). A survey on coverage path planning for robotics. Robotics and Autonomous Systems, 61, 1258-1276. doi:10.1016/j.robot.2013.09.004.

García-Heras, J., Soler, M., \& Sáez, F. J. (2014). A Comparison of Optimal Control Methods for Minimum Fuel Cruise at Constant Altitude and Course with Fixed Arrival Time. Procedia Engineering, 80, 231244. doi:10.1016/j.proeng.2014.09.083.

Gasparetto, A., Boscariol, P., Lanzutti, A., \& Vidoni, R. (2015). Path Planning and Trajectory Planning Algorithms: A General Overview. In Motion and Operation Planning of Robotic Systems Mechanisms and Machine Science (pp. 3-27). Springer, Cham. doi:10.1007/978-3-319-14705-5\_1.

Goddard, R. H. (1919). A method of reaching extreme altitudes (with 10 plates). Smithsonian Miscellaneous Collections, 71, 1-69.

Goerzen, C., Kong, Z., \& Mettler, B. (2009). A Survey of Motion Planning Algorithms from the Perspective of Autonomous UAV Guidance. Journal of Intelligent and Robotic Systems, 57, 65. doi:10.1007/s10846-009-9383-1.

Golden, B., Raghavan, S., \& Wasil, E. (Eds.) (2008). The Vehicle Routing Problem: Latest Advances and New Challenges volume 43 of Operations Research/Computer Science Interfaces. Boston, MA: Springer US.

Golden, B. L., \& Assad, A. (1988). Vehicle Routing: Methods and Studies. Amsterdam: North-Holland.

Gottlieb, Y., \& Shima, T. (2015). UAVs Task and Motion Planning in the Presence of Obstacles and Prioritized Targets. Sensors, 15, 29734-29764. doi:10.3390/s151129734. 
Guerriero, F., Surace, R., Loscrí, V., \& Natalizio, E. (2014). A multi-objective approach for unmanned aerial vehicle routing problem with soft time windows constraints. Applied Mathematical Modelling, 38, 839-852. doi:10.1016/j.apm.2013.07.002.

Ha, Q. M., Deville, Y., Pham, Q. D., \& Há, M. H. (2015). On the Min-cost Traveling Salesman Problem with Drone. Working Paper.

Han, D.-H., Kim, Y.-D., \& Lee, J.-Y. (2014). Multiple-criterion shortest path algorithms for global path planning of unmanned combat vehicles. Computers $\&$ Industrial Engineering, 71, 57-69. doi:10.1016/ j.cie.2014.02.013.

Hayat, S., Yanmaz, E., \& Muzaffar, R. (2016). Survey on Unmanned Aerial Vehicle Networks for Civil Applications: A Communications Viewpoint. IEEE Communications Surveys \& Tutorials, 18, 1-1. doi:10.1109/COMST.2016.2560343.

Henchey, M. J., Batta, R., Karwan, M., \& Crassidis, A. (2016). A Flight Time Approximation Model for Unmanned Aerial Vehicles. In J. R. C. C. (Ret.), US Navy, \& J. Q. D. Jr (Eds.), Operations Research for Unmanned Systems (pp. 95-117). John Wiley \& Sons, Ltd.

Ho, H.-M., \& Ouaknine, J. (2015). The Cyclic-Routing UAV Problem is PSPACE-Complete. In A. Pitts (Ed.), Foundations of Software Science and Computation Structures number 9034 in Lecture Notes in Computer Science (pp. 328-342). Springer Berlin Heidelberg. doi:10.1007/978-3-662-46678-0_21.

Hu, X., Cheng, J., \& Luo, H. (2015a). Task Assignment for Multi-UAV under Severe Uncertainty by Using Stochastic Multicriteria Acceptability Analysis. Mathematical Problems in Engineering, 2015, 1-10. doi:10.1155/2015/249825.

Hu, X., Ma, H., Ye, Q., \& Luo, H. (2015b). Hierarchical method of task assignment for multiple cooperating UAV teams. Journal of Systems Engineering and Electronics, 26, 1000-1009. doi:10.1109/JSEE.2015.00109.

Huang, L., Qu, H., Ji, P., Liu, X., \& Fan, Z. (2016). A novel coordinated path planning method using k-degree smoothing for multi-UAVs. Applied Soft Computing, 48, 182-192. doi:10.1016/ j.asoc.2016.06.046.

Jaishankar, S., \& Pralhad, R. N. (2011). 3d Off-Line Path Planning For Aerial Vehicle Using Distance Transform Technique. Procedia Computer Science, 4, 1306-1315. doi:10.1016/j.procs.2011.04.141.

Jiang, J., \& Ng, K. M. (2011). Priority-based routing of unmanned combat aerial vehicles. In Defense Science Research Conference and Expo (DSR), 2011 (pp. 1-4). doi:10.1109/DSR.2011.6026850.

Kagabo, W. (2010). Optimal trajectory planning for a UAV glider using atmospheric thermals. Msc. Thesis Rochester Institute of Technology Rochester, New York.

675 Kanistras, K., Martins, G., Rutherford, M., \& Valavanis, K. (2013). A survey of unmanned aerial vehicles (uavs) for traffic monitoring. In Unmanned Aircraft Systems (ICUAS), 2013 International Conference on (pp. 221-234). 
Karaman, S., \& Inalhan, G. (2008). Large-scale Task/Target Assignment for UAV Fleets Using a Distributed Branch and Price Optimization Scheme. In Proceedings of the 17th World Congress (pp. 13310-13317). volume 41 of 17th IFAC World Congress.

Keane, J. F., \& Carr, S. S. (2013). A Brief History of Early Unmanned Aircraft. Johns Hopkins APL Technical Digest, 32, 558-571.

Khamis, A., Hussein, A., \& Elmogy, A. (2015). Multi-robot Task Allocation: A Review of the Stateof-the-Art. In A. Koubâa, \& J. R. M.-d. Dios (Eds.), Cooperative Robots and Sensor Networks 2015 number 604 in Studies in Computational Intelligence (pp. 31-51). Springer International Publishing. doi:10.1007/978-3-319-18299-5_2.

Kim, M.-H., Baik, H., \& Lee, S. (2015). Resource Welfare Based Task Allocation for UAV Team with Resource Constraints. Journal of Intelligent E Robotic Systems, 77, 611-627. doi:10.1007/s10846014-0088-8.

Kim, Y., Gu, D.-W., \& Postlethwaite, I. (2007). Real-Time Optimal Time-Critical Target Assignment for UAVs. In P. M. Pardalos, R. Murphey, D. Grundel, \& M. J. Hirsch (Eds.), Advances in Cooperative Control and Optimization number 369 in Lecture Notes in Control and Information Sciences (pp. 265-280). Springer Berlin Heidelberg. doi:10.1007/978-3-540-74356-9_16.

Kingston, D. B., \& Schumacher, C. J. (2005). Time-dependent cooperative assignment. In ACC: Proceedings of the 2005 American Control Conference, Vols 1-7 (pp. 4084-4089). New York: IEEE.

Kirk, D. E. (2012). Optimal Control Theory: An Introduction. Courier Corporation.

Kivelevitch, E., Cohen, K., \& Kumar, M. (2016). Near-Optimal Assignment of UAVs to Targets Using a Market-Based Approach. In J. R. C. C. (Ret.), US Navy, \& J. Q. D. Jr (Eds.), Operations Research for Unmanned Systems (pp. 27-57). John Wiley \& Sons, Ltd.

Kumar, P., \& Padhi, R. (2013). Suboptimal Guidance of UAVs Satisfying Multiple Constraints on Waypoints. IFAC Proceedings Volumes, 46, 78-83. doi:10.3182/20130902-5-DE-2040.00112.

Kunchev, V., Jain, L., Ivancevic, V., \& Finn, A. (2006). Path Planning and Obstacle Avoidance for Autonomous Mobile Robots: A Review. In B. Gabrys, R. J. Howlett, \& L. C. Jain (Eds.), KnowledgeBased Intelligent Information and Engineering Systems number 4252 in Lecture Notes in Computer Science (pp. 537-544). Springer Berlin Heidelberg. doi:10.1007/11893004_70.

Kwak, D. J., Moon, S., Kim, S., \& Kim, H. J. (2013). Optimization of Decentralized Task Assignment for Heterogeneous UAVs. In Proceedings of the 11th IFAC Workshop on Adaptation and Learning in Control and Signal Processing (pp. 251-256). volume 46.

Lahyani, R., Khemakhem, M., \& Semet, F. (2015). Rich vehicle routing problems: From a taxonomy to a definition. European Journal of Operational Research, 241, 1-14. doi:10.1016/j.ejor.2014.07.048.

Latombe, J.-C. (1991). Introduction and Overview. In Robot Motion Planning number 124 in The Springer International Series in Engineering and Computer Science (pp. 1-57). Springer US. 
Levy, D., Sundar, K., \& Rathinam, S. (2014). Heuristics for Routing Heterogeneous Unmanned Vehicles with Fuel Constraints. Mathematical Problems in Engineering, 2014, 1-12. doi:10.1155/2014/131450.

Lin, L., Qibo, S., Shangguang, W., \& Fangchun, Y. (2013). Research on PSO Based Multiple UAVs Real-Time Task Assignment. In 2013 25th Chinese Control and Decision Conference (pp. 1530-1536). New York: IEEE.

Liu, H., Lin, M., \& Deng, L. (2016). UAV route planning for aerial photography under interval uncertainties. Optik - International Journal for Light and Electron Optics, 127, 9695-9700. doi:10.1016/j.ijleo.2016.06.117.

Liu, W., Zheng, Z., \& Cai, K.-Y. (2013). Bi-level programming based real-time path planning for unmanned aerial vehicles. Knowledge-Based Systems, 44, 34-47. doi:10.1016/j.knosys.2013.01.011.

Manyam, S. G., Rathinam, S., \& Darbha, S. (2015). Computation of Lower Bounds for a Multiple Depot, Multiple Vehicle Routing Problem With Motion Constraints. Journal of Dynamic Systems, Measurement, and Control, 137, 1-6. doi:10.1115/1.4030354.

Medeiros, A. C., \& Urrutia, S. (2010). Discrete optimization methods to determine trajectories for Dubins' vehicles. Electronic Notes in Discrete Mathematics, 36, 17-24. doi:10.1016/j.endm.2010.05.003.

Mersheeva, V. (2015). UAV Routing Problem for Area Monitoring in a Disaster Situation. Ph.D. Thesis Alpen-Adria-Universität Klagenfurt Austria.

Mufalli, F., Batta, R., \& Nagi, R. (2012). Simultaneous sensor selection and routing of unmanned aerial vehicles for complex mission plans. Computers \& Operations Research, 39, 2787-2799. doi:10.1016/ j.cor.2012.02.010.

Murray, C., \& Karwan, M. (2013). A branch-and-bound-based solution approach for dynamic rerouting of airborne platforms. Naval Research Logistics (NRL), 60, 141-159. doi:10.1002/nav.21526.

Murray, C. C., \& Chu, A. G. (2015). The flying sidekick traveling salesman problem: Optimization of drone-assisted parcel delivery. Transportation Research Part C: Emerging Technologies, 54, 86-109. doi:10.1016/j.trc.2015.03.005.

Murray, C. C., \& Karwan, M. H. (2010). An extensible modeling framework for dynamic reassignment and rerouting in cooperative airborne operations. Naval Research Logistics (NRL), 57, 634-652. doi:10.1002/nav.20427.

Myers, D., Batta, R., \& Karwan, M. (2016). A real-time network approach for including obstacles and flight dynamics in UAV route planning. The Journal of Defense Modeling and Simulation: Applications, Methodology, Technology, 13, 291-306. doi:10.1177/1548512916630183.

Nedjati, A., Vizvari, B., \& Izbirak, G. (2016). Post-earthquake response by small UAV helicopters. Natural Hazards, 80, 1669-1688. doi:10.1007/s11069-015-2046-6.

Nex, F., \& Remondino, F. (2013). UAV for 3d mapping applications: a review. Applied Geomatics, 6, 1-15. doi:10.1007/s12518-013-0120-x. 
Nguyen, J. L., Lawrance, N. R. J., Fitch, R., \& Sukkarieh, S. (2015). Real-time path planning for longterm information gathering with an aerial glider. Autonomous Robots, 40, 1-23. doi:10.1007/s10514015-9515-3.

Niccolini, M., Innocenti, M., \& Pollini, L. (2010). Multiple UAV Task Assignment using Descriptor Functions. IFAC Proceedings Volumes, 43, 93-98. doi:10.3182/20100906-5-JP-2022.00017.

NPD (2016). Year-Over-Year Drone Revenue Soars, According to NPD. https://www.npd.com/wps/ $\mathrm{portal} / \mathrm{npd} / \mathrm{us} / \mathrm{news} / \mathrm{press}-r e l e a s e s / 2016 /$ year-over-year-drone-revenue-soars-accordingto-npd/. Accessed: 15-10-2016.

Park, C.-H., Kim, Y.-D., \& Jeong, B. (2012). Heuristics for determining a patrol path of an unmanned combat vehicle. Computers \& Industrial Engineering, 63, 150-160. doi:10.1016/j.cie.2012.02.007.

Pepy, R., \& Hérissé, B. (2014). An Indirect Method for Optimal Guidance of a Glider. IFAC Proceedings Volumes, 47, 5097-5102. doi:10.3182/20140824-6-ZA-1003.01261.

Pharpatara, P., Hérissé, B., \& Bestaoui, Y. (2015). 3D-shortest paths for a hypersonic glider in a heterogeneous environment. IFAC-PapersOnLine, 48, 186-191. doi:10.1016/j.ifacol.2015.08.081.

Quaritsch, M., Kruggl, K., Wischounig-Strucl, D., Bhattacharya, S., Shah, M., \& Rinner, B. (2010). Networked UAVs as aerial sensor network for disaster management applications. Elektrotech. Inftech., 127, 56-63. doi:10.1016/j.ifacol.2015.08.081.

Raivio, T., Ehtamo, H., \& Hämäläinem, R. P. (1996). Aircraft trajectory optimization using nonlinear programming. In J. Dolezăl, \& J. Fidler (Eds.), System Modelling and Optimization IFIP: The International Federation for Information Processing (pp. 435-441). Springer US. doi:10.1007/978-0-38734897-1_52.

Ramirez-Atencia, C., Bello-Orgaz, G., R-Moreno, M. D., \& Camacho, D. (2016). Solving complex multiUAV mission planning problems using multi-objective genetic algorithms. Soft Computing, To appear, 1-18. doi:10.1007/s00500-016-2376-7.

Rao, A. V. (2014). Trajectory Optimization: A Survey. In H. Waschl, I. Kolmanovsky, M. Steinbuch, \& L. d. Re (Eds.), Optimization and Optimal Control in Automotive Systems number 455 in Lecture Notes in Control and Information Sciences (pp. 3-21). Springer International Publishing. doi:10.1007/ 978-3-319-05371-4_1.

Rao, B., Gopi, A. G., \& Maione, R. (2016). The societal impact of commercial drones. Technology in Society, 45, 83-90. doi:10.1016/j.techsoc.2016.02.009.

Rathinam, S., \& Sengupta, R. (2007). Algorithms for Routing Problems Involving UAVs. In D. J. S. Chahl, P. L. C. Jain, D. A. Mizutani, \& P. M. Sato-Ilic (Eds.), Innovations in Intelligent Machines - 1 number 70 in Studies in Computational Intelligence (pp. 147-172). Springer Berlin Heidelberg. doi:10.1007/978-3-540-72696-8_6. 
Reif, J., \& Sharir, M. (1994). Motion Planning in the Presence of Moving Obstacles. J. ACM, 41, 764-790. doi:10.1145/179812.179911.

Rogowski, K., \& Maroński, R. (2011). Optimization of glider's trajectory for given thermal conditions. Archive of Mechanical Engineering, 58, 11-25. doi:10.2478/v10180-011-0001-y.

Ross, I. M. (2009). A Primer on Pontryagin's Principle in Optimal Control. Collegiate Publishers.

Ruzgiené, B., Berteška, T., Gečyte, S., Jakubauskiené, E., \& Aksamitauskas, V. C̆. (2015). The surface modelling based on UAV Photogrammetry and qualitative estimation. Measurement, 73, 619-627. doi:https://doi.org/10.1016/j.measurement.2015.04.018.

Rysdyk, R. (2006). Unmanned Aerial Vehicle Path Following for Target Observation in Wind. Journal of Guidance, Control, and Dynamics, 29, 1092-1100. doi:10.2514/1.19101.

Schultz, R. L., \& Zagalsky, N. R. (1972). Aircraft performance optimization. Journal of Aircraft, 9, 108-114. doi:10.2514/3.44326.

Shanmugavel, M. (2013). Dynamic risk uncertainties by cooperative sites in path planning of UAVs. IFAC Proceedings Volumes, 46, 310-315. doi:10.3182/20131120-3-FR-4045.00023.

Shima, T., \& Schumacher, C. (2009). Assigning cooperating UAVs to simultaneous tasks on consecutive targets using genetic algorithms. Journal of the Operational Research Society, 60, 973-982. doi:10.1057/palgrave.jors.2602617.

Silva, W., Frew, E. W., \& Shaw-Cortez, W. (2015). Implementing path planning and guidance layers for dynamic soaring and persistence missions. In 2015 International Conference on Unmanned Aircraft Systems (ICUAS) (pp. 92-101).

Song, B. D., Kim, J., \& Morrison, J. R. (2016). Rolling horizon path planning of an autonomous system of uavs for persistent cooperative service: MILP formulation and efficient heuristics. Journal of Intelligent E6 Robotic Systems, 84, 241-258. doi:10.1007/s10846-015-0280-5.

Stengel, R. F. (2004). Flight Dynamics. Princeton University Press.

Stöcker, C., Eltner, A., \& Karrasch, P. (2015). Measuring gullies by synergetic application of UAV and close range photogrammetry: A case study from Andalusia, Spain. Catena, 132, 1-11. doi:10.1016/ j.catena.2015.04.004.

Stryk, O. v., \& Bulirsch, R. (1992). Direct and indirect methods for trajectory optimization. Annals of Operations Research, 37, 357-373. doi:10.1007/BF02071065.

Stump, E., \& Michael, N. (2011). Multi-robot persistent surveillance planning as a Vehicle Routing Problem. In 2011 IEEE Conference on Automation Science and Engineering (CASE) (pp. 569-575). doi:10.1109/CASE.2011.6042503.

Sundar, K., \& Rathinam, S. (2014). Algorithms for Routing an Unmanned Aerial Vehicle in the Presence of Refueling Depots. IEEE Transactions on Automation Science and Engineering, 11, $287-294$. doi:10.1109/TASE.2013.2279544. 
Techy, L., Woolsey, C. A., \& Morgansen, K. A. (2010). Planar path planning for flight vehicles in wind with turn rate and acceleration bounds. In 2010 IEEE International Conference on Robotics and Automation (ICRA) (pp. 3240-3245). doi:10.1109/ROBOT.2010.5509862.

Vilar, R. G., \& Shin, H.-S. (2013). Communication-Aware Task Assignment for UAV Cooperation in Urban Environments. In Proceedings of the 2nd IFAC Workshop on Research, Education and Development of Unmanned Aerial Systems (pp. 352-359). volume 46.

Wang, G.-G., Chu, H. E., \& Mirjalili, S. (2016). Three-dimensional path planning for UCAV using an improved bat algorithm. Aerospace Science and Technology, 49, 231-238. doi:10.1016/j.ast.2015.11.040.

Wang, J., Zhang, Y. F., Geng, L., Fuh, J. Y. H., \& Teo, S. H. (2015). A Heuristic Mission Planning Algorithm for Heterogeneous Tasks with Heterogeneous UAVs. Unmanned Systems, 03, 205-219. doi:10.1142/S2301385015500132.

Wang, X. (2009). Solving optimal control problems with MATLAB: Indirect methods. Technical Report ISE. Dept., NCSU, Raleigh, NC 27695.

Wang, X., Poikonen, S., \& Golden, B. (2017). The vehicle routing problem with drones: several worst-case results. Optimization Letters, 11, 679-697. doi:10.1007/s11590-016-1035-3.

Wu, J.-P., Peng, Z.-H., \& Chen, J. (2011). 3d Multi-Constraint Route Planning for UAV Low-altitude Penetration Based on Multi-Agent Genetic Algorithm. IFAC Proceedings Volumes, 44, 11821-11826. doi:10.3182/20110828-6-IT-1002.01770

Xu, S., Doğançay, K., \& Hmam, H. (2017). Distributed pseudolinear estimation and UAV path optimization for 3d AOA target tracking. Signal Processing, 133, 64-78. doi:10.1016/j.sigpro.2016.10.012.

Xu, Z., Yang, J., Peng, C., Wu, Y., Jiang, X., Li, R., Zheng, Y., Gao, Y., Liu, S., \& Tian, B. (2014). Development of an UAS for post-earthquake disaster surveying and its application in ms7.0 lushan earthquake, sichuan, china. Computers $\& 3$ Geosciences, 68, 22-30. doi:10.1016/j.cageo.2014.04.001. 
Yakıcı, E. (2016). Solving Location and Routing Problem for UAVs. Computers \& Industrial Engineering, 102, 294-301. doi:10.1016/j.cie.2016.10.029.

Yang, L., Qi, J., Song, D., Xiao, J., Han, J., \& Xia, Y. (2016). Survey of Robot 3d Path Planning Algorithms. Journal of Control Science and Engineering, 2016, 1-22. doi:10.1155/2016/7426913.

Yang, Y., Karimadini, M., Xiang, C., Teo, S. H., Chen, B. M., \& Lee, T. H. (2015). Wide area surveillance of urban environments using multiple Mini-VTOL UAVs. In IECON 2015 - 41st Annual Conference of the IEEE Industrial Electronics Society (pp. 795-800). doi:10.1109/IECON.2015.7392196.

Yomchinda, T., Horn, J. F., \& Langelaan, J. W. (2016). Modified Dubins parameterization for aircraft emergency trajectory planning. Journal of Aerospace Engineering, 1, 1-20. doi:10.1177/ 0954410016638869.

YongBo, C., YueSong, M., JianQiao, Y., XiaoLong, S., \& Nuo, X. (2017). Three-dimensional unmanned aerial vehicle path planning using modified wolf pack search algorithm. Neurocomputing, 266, 445457. doi:https://doi.org/10.1016/j.neucom.2017.05.059.

Yuan, C., Zhang, Y., \& Liu, Z. (2015). A survey on technologies for automatic forest fire monitoring, detection, and fighting using unmanned aerial vehicles and remote sensing techniques. Can. J. For. Res., 45, 783-792. doi:10.1139/cjfr-2014-0347.

Zhang, X., Chen, J., Xin, B., \& Fang, H. (2011). Online Path Planning for UAV Using an Improved Differential Evolution Algorithm. IFAC Proceedings Volumes, 44, 6349-6354. doi:10.3182/201108286-IT-1002.01807.

Zhang, X., Chen, J., Xin, B., \& Peng, Z. (2014). A memetic algorithm for path planning of curvatureconstrained UAVs performing surveillance of multiple ground targets. Chinese Journal of Aeronautics, 27, 622-633. doi:10.1016/j.cja.2014.04.024.

Zhang, Y., Chen, J., \& Shen, L. (2012). Hybrid hierarchical trajectory planning for a fixed-wing UCAV performing air-to-surface multi-target attack. Journal of Systems Engineering and Electronics, 23, 536-552. doi:10.1109/JSEE.2012.00068. 